\title{
Rapidly rotating cylinder flow with an oscillating sidewall
}

\author{
Juan M. Lopez ${ }^{*}$ \\ School of Mathematical and Statistical Sciences, Arizona State University, Tempe, Arizona 85287, USA \\ Francisco Marques \\ Department of Física Aplicada, Universitat Politècnica de Catalanya, Girona s/n, Mòdul B4 Campus Nord, 08034 Barcelona, Spain
}

(Received 4 September 2013; revised manuscript received 29 November 2013; published 17 January 2014)

\begin{abstract}
We present numerical simulations of a flow in a rapidly rotating cylinder subjected to a time-periodic forcing via axial oscillations of the sidewall. When the axial oscillation frequency is less than twice the rotation frequency, inertial waves in the form of shear layers are present. For very fast rotations, these waves approach the form of the characteristics predicted from the linearized inviscid problem first studied by Lord Kelvin. The driving mechanism for the inertial waves is the oscillating Stokes layer on the sidewall and the corner discontinuities where the sidewall meets the top and bottom end walls. A detailed numerical and theoretical analysis of the internal shear layers is presented. The system is physically realizable, and attractive because of the robustness of the Stokes layer that drives the inertial waves but beyond that does not interfere with them. We show that the system loses stability to complicated three-dimensional flow when the sidewall oscillation displacement amplitude is very large (of the order of the cylinder radius), but this is far removed from the displacement amplitudes of interest, and there is a large range of governing parameters which are physically realizable in experiments in which the inertial waves are robust. This is in contrast to many other physical realizations of inertial waves where the driving mechanisms tend to lead to instabilities and complicate the study of the waves. We have computed the response diagram of the system for a large range of forcing frequencies and compared the results with inviscid eigenmodes and ray tracing techniques.
\end{abstract}

DOI: 10.1103/PhysRevE.89.013013

PACS number(s): 47.32.Ef, 47.35.De

\section{INTRODUCTION}

Rapidly rotating flows have long been a source of fascination due to their ability to support waves with peculiar properties. Inertial waves in rapidly rotating flows are ubiquitous [1-4], they are present in some geophysical and astrophysical flows [5-8], and may contribute in dynamo action [5,9-13]; they also contribute to instabilities in military payloads [14-16]. The study of inertial waves dates back to Lord Kelvin [17].

Inertial waves arise in rotating fluids owing to the presence of Coriolis-type restoring forces [1,3,17,18]. For rapidly rotating fluids (where the rotation period is much smaller than the viscous diffusion time scale), the inviscid limit of the equations of motion for an infinitesimal disturbance to a background solid-body rotation reduces to a linear partial differential equation of hyperbolic type, provided the disturbance frequency is less than twice the background rotation frequency, and inertial waves may be excited through direct forcing. Most theoretical investigations of the inertial wave problem are inviscid, based on the free-slip (zero penetration) boundary conditions and the inviscid internal wave equation within the fluid. Simple geometries were studied first, namely circular or elliptical cylinders and spheres. Such inviscid approaches provide the radiated energy but not the wave profiles. For the latter, $a$ posteriori addition of the viscosity is necessary, although the addition is not fully consistent in that the effect of viscosity is taken into account on the propagation of the waves (in the wave equation) but not on their generation (in the boundary condition) [1,19-21]. This approximation implies that only

\footnotetext{
*jmlopez@asu.edu
}

the waves are retained while the other two components of the motion are neglected: the boundary layers and the local wave forcing within the fluid. In order to obtain all three components, explicit consideration of the no-slip condition is required.

Forced rapidly rotating flows manifest wave beams. These are the persistent responses to spatially localized periodic forcings. These localized forcings may be due to intrinsic boundary layer instabilities [22], boundary layer eruptions [23], or discontinuities in the boundary conditions (such as in the present problem). In the inviscid setting, the characteristics of the wave equation would emanate from these locations at directions dictated by the dispersion relation. Viscosity regularizes the discontinuities corresponding to the characteristics and they manifest as internal shear layers (wave beams) that are also called inertial waves, and the associated internal reflections of wave characteristics are clearly observable in suitably designed experiments [1]. They are analogous to internal waves in linearly stratified flows, iconically identified with St. Andrew's cross.

The experimental work on inertial waves so far suggests that inertial waves are generically unstable above a threshold amplitude. However, we note that, in all such experiments, either the geometry has been subjected to significant distortions, such as the "squeezed" rotating cylinders and spheres [24-26], or the rotation vector has been subjected to significant distortions in either its direction, such as in precessions [6,16,27-33], or its magnitude, such as in librations [8,34,35]. Typically, theoretical approaches have not and are not analytically capable of taking these distortions into account, and the distortions are prone to introduce instabilities that complicate the analysis. We have chosen in the present study a robust way of generating inertial waves, based on an oscillatory Stokes flow: axial oscillations of the sidewall of a rotating cylinder. 
Direct numerical simulations of the full problem (NavierStokes equations with very small Ekman number and no-slip boundary conditions) are few and far between, especially for fully three-dimensional simulations. Early attempts at full direct numerical simulations [21] were extremely limited in what they could resolve; the thin boundary layers caused severe problems, and subsequent attempts utilized stress-free boundary conditions to avoid the challenge of resolving the viscous boundary layers [36,37]. This approach, however, is unsatisfactory as there is the real danger of rejecting the essential along with the inessential. We have seen in rapidly rotating flow problems driven by steady differential rotation (so that inertial waves are not driven directly by the forcing) that the thin viscous boundary layers can become unstable and the associated temporal frequencies then drive inertial waves that transmit energy and shear deep into the interior that is nominally in rapid solid-body rotation $[22,38,39]$. It is crucial to take into account the effects of confinement in rotating flows.

We have solved the rapidly rotating cylinder flow with oscillating sidewall by using no-slip boundary conditions and an accurate numerical spectral method. We have found that the corner vortex emanating from the discontinuity of the boundary conditions is responsible for the formation of inertial wave beams that are stable and robust in a large parameter region, allowing for a detailed study of the inertial wave properties.

The paper begins with a description of the physical setting, the governing equations, boundary conditions and nondimensional governing parameters, and the numerical solution method used in Sec. II. In Sec. III, the Navier-Stokes equations solutions for a frequency that produces an inertial wave beam that starts at one corner, reflects on the axis at midheight, and ends at the other corner are examined over a wide range of rotation rates of the cylinder, giving asymptotic estimates of the width and intensity of the wave beams. The stability of these solutions is determined in Sec. III A, and in Sec. III B the inertial wave beam solutions from the Navier-Stokes simulations over a wide range of forcing frequencies are compared with inviscid eigenmodes and ray tracing. Finally, Sec. IV summarizes the results and draws conclusions.

\section{GOVERNING EQUATIONS AND NUMERICAL METHODS}

Consider the flow in a circular cylinder of radius $a$ and height $h$, completely filled with a fluid of kinematic viscosity $\nu$, rotating with an angular speed $\omega$, and whose sidewall oscillates in the axial direction with angular frequency $\omega_{f}$ and maximum axial displacement $\delta h$; in dimensional variables, $z^{*}(t)=\delta h \sin \left(\omega_{f} t^{*}\right)$, and the maximum speed of the wall is $\delta h \omega_{f}$. A schematic of the flow system is shown in Fig. 1. The Navier-Stokes equations, nondimensionalized using $a$ as the length scale and the viscous time $a^{2} / v$ as the time scale, and written in the rotating reference frame, are

$$
\left(\partial_{t}+\boldsymbol{u} \cdot \nabla\right) \boldsymbol{u}=-\nabla p-2 \boldsymbol{\Omega} \times \boldsymbol{u}+\nabla^{2} \boldsymbol{u}, \quad \boldsymbol{\nabla} \cdot \boldsymbol{u}=0,
$$

where $\boldsymbol{u}=(u, v, w)$ is the velocity field in polar coordinates $(r, \theta, z) \in[0,1] \times[0,2 \pi] \times[-\Gamma / 2, \Gamma / 2], p$ is the kinematic pressure, and $\boldsymbol{\Omega}=\Omega \boldsymbol{e}_{z}$ is the rotation vector pointing in the

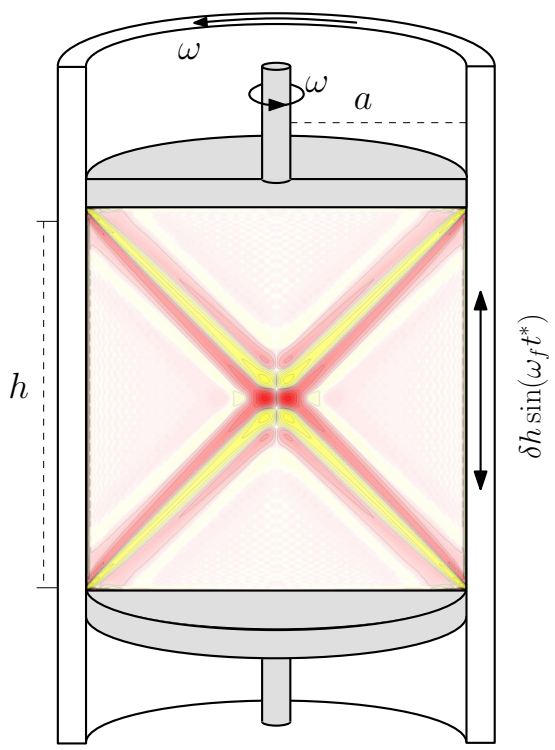

FIG. 1. (Color online) Schematic of the apparatus.

axial direction. There are four governing parameters:

$$
\begin{aligned}
\text { aspect ratio : } & \Gamma=h / a, \\
\text { rotating frequency : } & \Omega=\omega a^{2} / \nu, \\
\text { oscillation frequency : } & \Omega_{f}=\omega_{f} a^{2} / \nu, \\
\text { oscillation amplitude : } & \operatorname{Re}=\delta h \omega_{f} a / \nu .
\end{aligned}
$$

The boundary conditions are no slip:

$$
\text { sidewall, } r=1: \quad(u, v, w)=\left[0,0, \operatorname{Re} \cos \left(\Omega_{f} t\right)\right],
$$

top and bottom end walls, $z= \pm \Gamma / 2: \quad(u, v, w)=(0,0,0)$.

The frequency ratio $F=\Omega_{f} / 2 \Omega$ is of great relevance, as generally when it is less than one and $\Omega$ is sufficiently large, the system supports inertial waves. The movement of the sidewall is given by $z(t)=\delta h / a \sin \left(\Omega_{f} t\right)$. The maximum excursion of the sidewall relative to the cylinder radius is $\delta h / a=\operatorname{Re} / \Omega_{f}$. The Ekman number is often used to characterize viscous rotating flows. In terms of our parameters, the Ekman number is $E k=\Omega^{-1} \Gamma^{-2}$. We can also define a Rossby number to measure the relative importance of Coriolis to inertia as $\operatorname{Ro}=\operatorname{Re} / \Omega$. For the study of inertial waves, these two ratios should both be small, so that the flow is rotation dominated and subjected to small perturbations. In terms of the Rossby number, most of the results presented here are for $\operatorname{Re}=10^{2}$ and $\Omega=10^{5}$, giving $\operatorname{Ro}=10^{-3}$, where the flow is rotation dominated. For fixed $\operatorname{Re}=10^{2}$, we have also considered smaller $\Omega$ and find that the dynamics are rotation dominated down to $\Omega \sim 10^{3}$, corresponding to $\operatorname{Ro}=0.1$. Also, for $\Omega=10^{5}$, inertia effects from the sidewall oscillation become important at about $\operatorname{Re}=10^{4}$, again corresponding to Ro $=0.1$.

The governing equations (1) have been solved using a second-order time-splitting method, with space discretized via a Galerkin-Fourier expansion in $\theta$ and Chebyshev collocation 
(a)

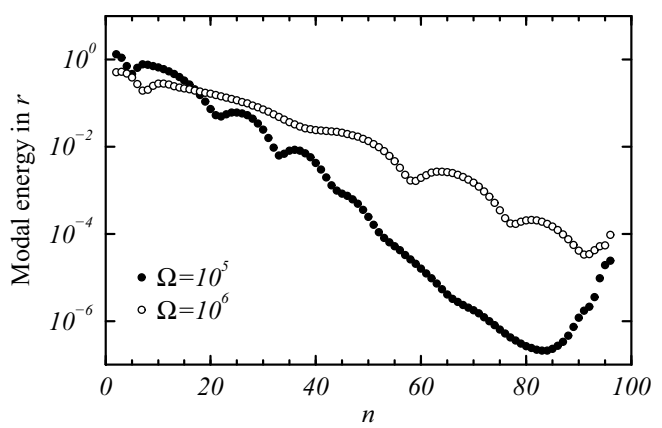

(b)

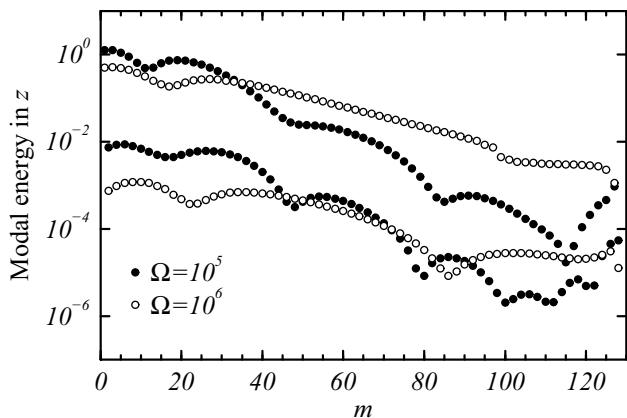

(c)

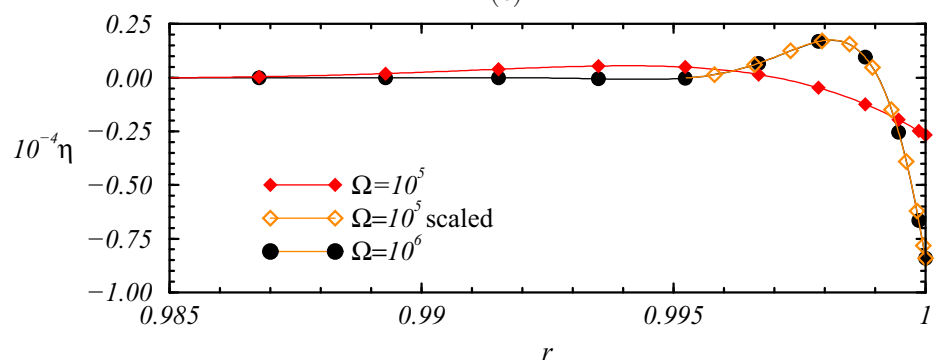

FIG. 2. (Color online) Spectral resolution in the (a) radial and (b) axial directions, and (c) the sidewall boundary layer profile of the azimuthal vorticity at the cylinder half-height at a phase where the displacement of the sidewall is maximum in the negative- $z$ direction which corresponds to maximum shear at the sidewall, all for $\operatorname{Re}=10^{2}, F=0.7071068$, and $\Omega$ as indicated.

in $r$ and $z$ :

$\boldsymbol{u}(r, \theta, z, t)=\sum_{n=0}^{2 n_{r}+1} \sum_{m=0}^{n_{z}} \sum_{k=-n_{\theta} / 2}^{k=n_{\theta} / 2-1} \hat{\boldsymbol{u}}_{m n k}(t) \boldsymbol{\Xi}_{n}(r) \boldsymbol{\Xi}_{m}(z) e^{i k \theta}$,

where $\Xi_{n}$ is the $n$th Chebyshev polynomial. The spectral solver is based on that described in Ref. [40] and it has been used extensively in a wide variety of enclosed cylinder flows. For the solutions presented here, with $\Gamma=2$, we have used up to $n_{r}=96$ and $n_{z}=128$ Chebyshev modes in the radial and axial directions. In this paper, we have mainly considered axisymmetric cases. Typically, we have used between 1000 and 5000 time steps per modulation period, $T=2 \pi / \Omega_{f}$, depending on the parameter regime.

The kinetic energies associated with each Chebyshev mode in the radial and axial directions, $n$ and $m$, provide a good measure of the spectral resolution of the numerical solutions. For axisymmetric flows, the azimuthal Fourier mode $k=0$ and $\hat{\boldsymbol{u}}_{m n k}(t)$ is real. The velocity field can be separated into its radial and axial components:

$$
\begin{aligned}
\boldsymbol{u}_{n} & =\sum_{m=0}^{n_{z}} \hat{\boldsymbol{u}}_{m n 0}(t) \boldsymbol{\Xi}_{n}(r) \Xi_{m}(z) \quad \text { and } \\
\boldsymbol{u}_{m} & =\sum_{n=0}^{2 n_{r}+1} \hat{\boldsymbol{u}}_{m n 0}(t) \boldsymbol{\Xi}_{n}(r) \Xi_{m}(z),
\end{aligned}
$$

and the corresponding modal kinetic energies are

$$
\begin{aligned}
\mathrm{KE}_{m} & =\frac{1}{2} \int_{-0.5 \Gamma}^{0.5 \Gamma} \int_{0}^{1} \boldsymbol{u}_{m}^{2} r d r d z \quad \text { and } \\
\mathrm{KE}_{n} & =\frac{1}{2} \int_{-0.5 \Gamma}^{0.5 \Gamma} \int_{0}^{1} u_{n}^{2} r d r d z .
\end{aligned}
$$

Figures 2(a) and 2(b) show spectral convergence results for the typical case of $\Omega=10^{5}$ and the most demanding case computed, $\Omega=10^{6}$ (corresponding to $\mathrm{Ek}=2.5 \times 10^{-7}$ ), giving a global measure of how well resolved the flows are. The kinetic energies shown are in the rotating frame as in the stationary frame the $n=0, n=1$, and $m=0$ kinetic energies, dominated by the solid-body rotation, are of order $\Omega$ and hence are several orders of magnitude larger than the other modal energies; in this way the decay in the higher modes may be more readily appreciated. For $\Omega \leqslant 10^{5}$, the spectral modes decay by at least four orders of magnitude, giving well-resolved solutions to four significant figures. In the most demanding case computed, $\Omega=10^{6}$, the spectral coefficients still decay by two and three orders of magnitude for the axial and radial modes, sufficient for a good description of the flow. Another notable feature is that the even axial modes of the depicted solutions are much smaller than the odd modes due to the flow structure and symmetries (discussed in Sec. III), so in Fig. 2(b) it seems as if there are two different curves for the axial modes in each of the two cases analyzed.

The finest feature of the flows is the Stokes layer on the oscillating sidewall in the high-frequency regime associated with large $\Omega$. Figure 2(c) shows the sidewall boundary layer profiles of the azimuthal vorticity at the cylinder half-height at a phase where the displacement of the sidewall is maximum in the negative- $z$ direction, which corresponds to maximum shear at the sidewall, for $\operatorname{Re}=10^{2}, F=0.7071068, \Gamma=2$, and $\Omega=10^{5}$ (the case shown in Fig. 1) and $\Omega=10^{6}$ (the largest $\Omega$ considered), both solved using $n_{r}=96$ and $n_{z}=128$. In the $\Omega=10^{6}$ case, the boundary layer thickness is about 0.003 and there are six collocation points resolving it. Most of the results focus on the $\Omega=10^{5}$ case, whose sidewall boundary layer is about three times thicker and it is resolved with 10 
(a) $\Omega=10^{1}$

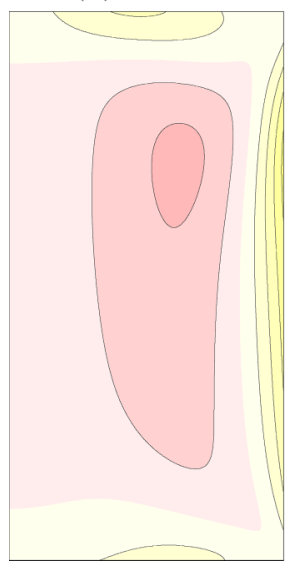

(b) $\Omega=10^{2}$

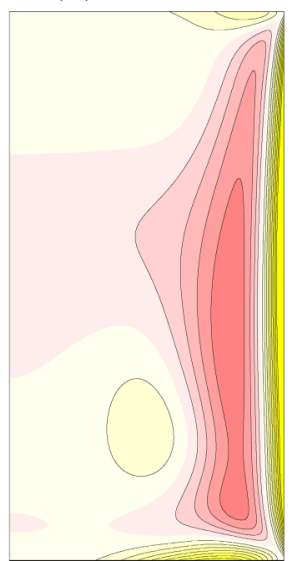

(c) $\Omega=10^{3}$

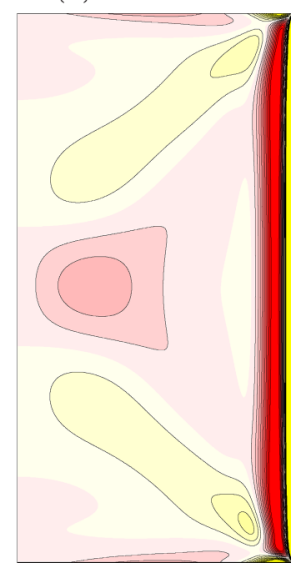

(d) $\Omega=10^{4}$

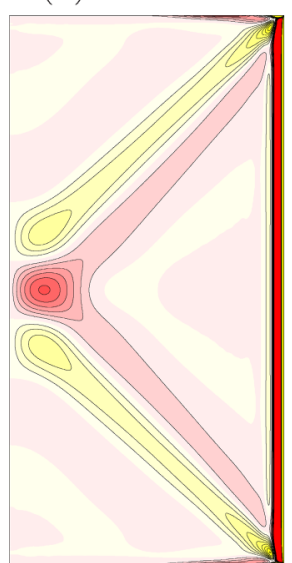

(e) $\Omega=10^{5}$

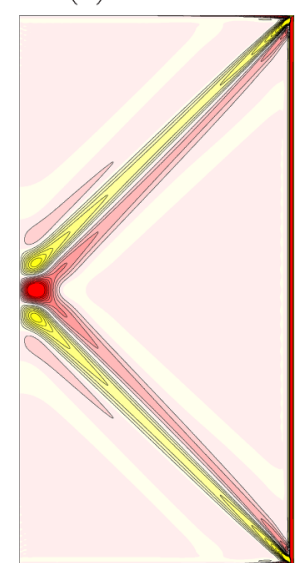

(f) $\Omega=10^{6}$

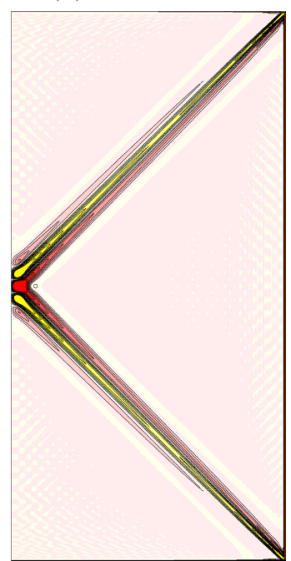

FIG. 3. (Color online) Contours of the azimuthal vorticity for $\operatorname{Re}=10^{2}, \Gamma=2, F=0.7071068$, and $\Omega$ as indicated, at a phase where the displacement of the sidewall is maximum in the negative- $z$ direction. There are 10 contour levels in the range $\eta \in[-500,500]$. For (a) $-(\mathrm{d})$ $n_{r} \times n_{z}=48 \times 96$ and $\delta t=T / 1000$, and for (e) and (f) $n_{r} \times n_{z}=96 \times 128$ and $\delta t=T / 5000$.

collocation points. The two boundary layer profiles shown are similar; scaling the radial distance from the wall by $1 / \sqrt{10}$ and $\eta$ by $\sqrt{10}$ for the $\Omega=10^{5}$ case (where 10 is the ratio of the value of $\Omega$ for the two cases) maps the $\Omega=10^{5}$ profile on top of the $\Omega=10^{6}$ profile, as is to be expected from the $\sqrt{\Omega}$ scaling for Stokes oscillatory boundary layers.

\section{A. Symmetries}

The governing equations and boundary conditions are invariant under arbitrary rotations through angle $\phi$ about the axis, $R_{\phi}$, whose action is

$$
R_{\phi}(u, v, w)(r, \theta, z, t)=(u, v, w)(r, \theta+\phi, z, t) .
$$

They are also invariant to the half-period-flip space-time symmetry corresponding to a half-forcing-period advance in time composed with a reflection about the cylinder half-height. The action $H$ of this symmetry is

$$
H(u, v, w)(r, \theta, z, t)=(u, v,-w)(r, \theta,-z, t+0.5 T) .
$$

The symmetry group of the system is $\mathcal{G}=\mathrm{SO}(2) \times Z_{2}$, with SO(2) generated by $R_{\phi}$ and $Z_{2}$ by $H$.

\section{III. $F<1$ FORCING AND INERTIAL WAVES}

The study of inertial waves dates back to Lord Kelvin [17] who noted that a fluid in solid-body rotation can sustain waves if their frequency $\sigma$ is less than twice the background rotation rate. The dispersion relation of inertial waves $[1,3]$ is

$$
\cos \beta=\sigma / 2 \omega,
$$

where $\beta \in[0,2 \pi)$ is the angle between the rotation vector and the wave vector, or in our case the angle between the horizontal plane (orthogonal to the cylinder axis) and the direction of the wave beam. Inertial waves are rapidly damped due to viscosity [19]. The only way to maintain them is using some external forcing. When a rotating fluid is forced continuously in time at a particular frequency, only inertial waves with the same frequency as the forcing are excited. In our periodically forced problem, the dispersion relation results in $\cos \beta=F$. For $F<1$ the system supports inertial waves if $\Omega$ is sufficiently large; here we address how large it needs to be. We consider the most distinctive inertial wave in the $\Gamma=2$ cylinder corresponding to $F=1 / \sqrt{2} \approx 0.7071068$. This corresponds to a characteristic in the $\Omega \rightarrow \infty$ limit that starts from one corner, reflects at the axis at midheight, and terminates at the opposite corner. Figure 3 shows contours of the azimuthal vorticity $\eta$ at a phase corresponding to the maximum displacement of the sidewall in the negative- $z$ direction for various $\Omega \in\left[10,10^{6}\right]$ and $\operatorname{Re}=10^{2}$. For small $\Omega<10^{3}$, the flow is very much like the $\Omega=0$ problem of [41] with a large roller being produced for each corner alternately as the sidewall oscillates up and down. The flow has the half-period-flip spatiotemporal symmetry (8). By $\Omega \approx 10^{3}$, the nature of the flow changes considerably. Instead of alternate rollers, we now have fairly well-defined shear layers emanating from one corner and entering the opposite corner, alternating in direction every half period, following the corresponding $\Omega \rightarrow \infty$ characteristics fairly well. As $\Omega$ is increased, the shear layers become much more sharply defined, as evidenced from Fig. 3(f) at the largest considered value of $\Omega=10^{6}$.

These changes in behavior correspond to the flow appearing to become more symmetric. The additional symmetry that seems apparent in Fig. 3 for $\Omega \gtrsim 10^{3}$ looks like the reflection about the midplane $z=0$; however, the azimuthal vorticity should change sign in such a reflection and it does not change. To better illustrate the flow and its symmetries, Fig. 4 shows for the $\Omega=10^{5}$ case contours of azimuthal velocity and vorticity, along with arrows corresponding to the projection of the velocity field in the $(r, z)$ plane. There are four plots equispaced over one forcing period $T$. The velocity of the sidewall is indicated by a single arrow at the sidewall; this provides a scale for the velocity vectors of the flow. Movies showing animations over one period are available online (see Supplemental Material [42]). From this figure (and movies), we also see that the three components of the velocity behave opposite to what should be expected in a midplane symmetry. What we appear to observe is a different symmetry $K$, whose actions on the velocity and vorticity 
$T / 4$

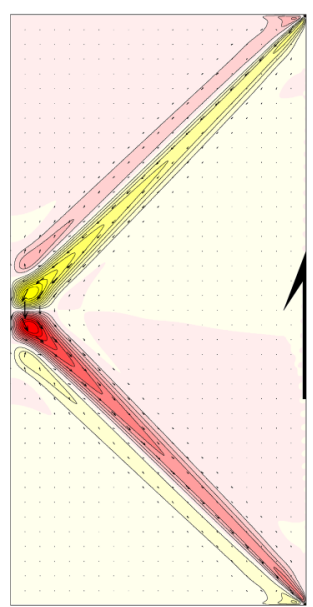

$3 T / 4$

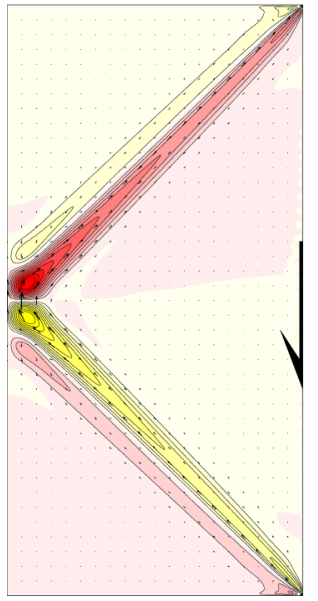

Azimuthal velocity $v$
$T / 2$

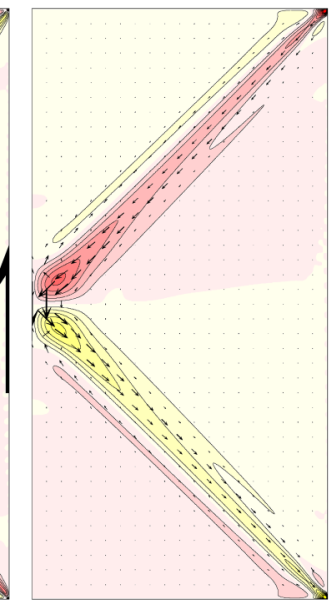

$T$

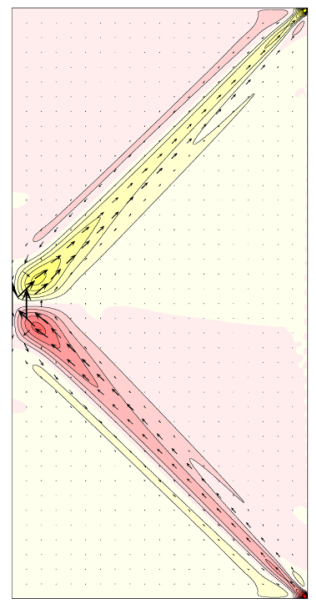

$T / 4$

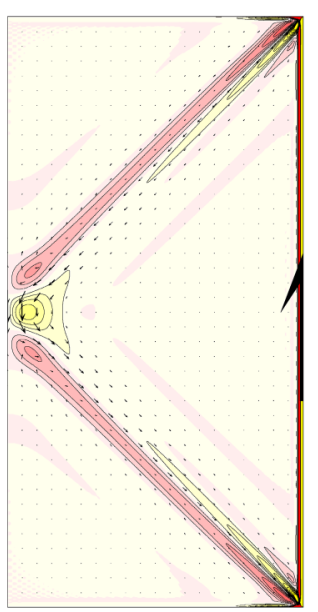

$3 T / 4$

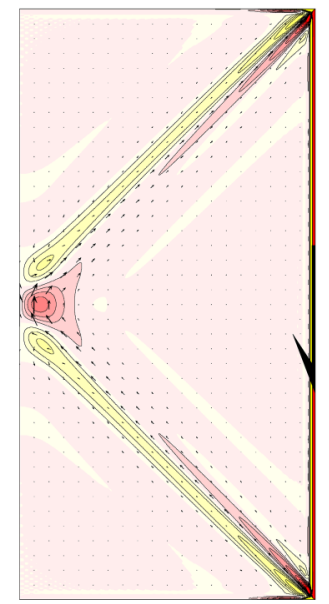

Azimuthal vorticity $\eta$
$T / 2$

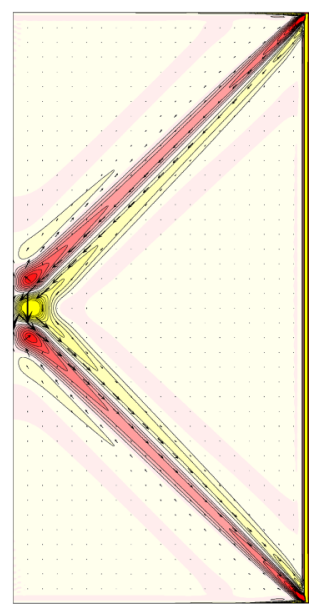

$T$

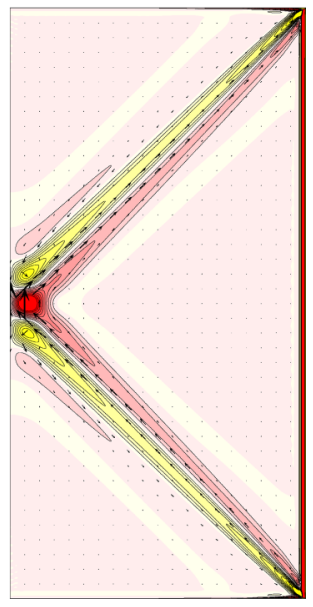

FIG. 4. (Color online) Contours of the azimuthal velocity and vorticity for $\Omega=10^{5}$, $\operatorname{Re}=10^{2}, \Gamma=2$, and $F=0.7071068$ at four phases during one period (there are ten contours in the ranges $v \in[-15,15], \eta \in[-500,500])$, together with vectors of the velocity field in the $(r, z)$ plane (only one vector is drawn at the sidewall indicating its velocity). A pair of movies showing the azimuthal velocity and vorticity over one period is available online [42].

are

$$
\begin{array}{r}
K(u, v, w)(r, \theta, z, t)=(-u,-v, w)(r, \theta,-z, t), \\
K(\xi, \eta, \zeta)(r, \theta, z, t)=(\xi, \eta,-\zeta)(r, \theta,-z, t) .
\end{array}
$$

However, $K$ is not a symmetry of the original problem $(1,2$, and 3), because the Navier-Stokes equations (1) are not equivariant under $K$ : the nonlinear and linear terms behave differently. So what is the origin of the different apparent symmetry? The inviscid linearized equations for the inertial waves and the corresponding nonpenetrating boundary conditions

$$
\text { sidewall, } r=1: \quad u=0,
$$

top and bottom end walls, $z= \pm 0.5 \Gamma: \quad w=0$,

are preserved under the actions of $K$. The flow tends to recover this symmetry $K$ of the inviscid linearized problem as $\Omega$ increases. This is because the boundary layer flow and the bulk flow (in the limit of an infinitely long cylinder) are invariant under $K . K$ is not a symmetry of the complete problem; the only symmetry, besides axisymmetry, is the space-time symmetry $H(8)$ that is preserved in our solutions, as can be seen from Fig. 4. But the $K$ symmetry preserves the boundary condition at the sidewall, (2), which is the driving mechanism of this problem; the only terms in the full problem that are not preserved by $K$ are the nonlinear terms in the Navier-Stokes equations. So, we can expect that the symmetry $K$ is approximately fulfilled so long as the amplitude of the forcing Re is not too large. This is precisely what is observed; there are very small differences between the top and bottom corner flows and in the boundary layers. These are barely visible in the solution at $\operatorname{Re}=10^{2}$ shown in Fig. 4. For larger Re the differences between the top and bottom corner flows become noticeable (this is discussed further below). The approximate fulfillment of the $K$ symmetry can be seen in Fig. 2(b), showing that the 


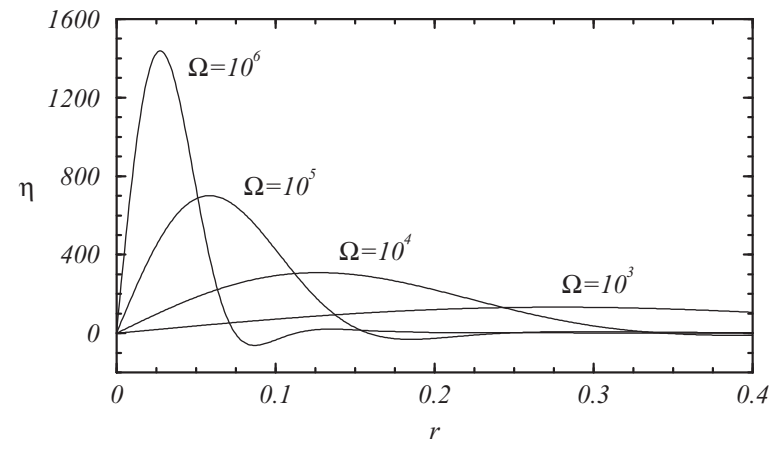

FIG. 5. Radial profiles of $\eta$ at midheight near the axis for $\mathrm{Re}=$ $10^{2}, \Gamma=2, F=0.7071068$, and $\Omega$ as indicated.

even spectral axial modes of the solutions are much smaller (by about two orders of magnitude) than the odd modes; if the $K$ symmetry were exact, the even modes should be zero.

The shear layer structure displayed in Fig. 4 has the following properties: along the straight shear layers extending from the corners to the center of the domain, the velocity field is orthogonal to the wave vector, the wave is circularly polarized, and the group velocity points from the corner towards the cylinder's geometric center, according to the selected forcing frequency, $\Omega_{f}=\sqrt{2} \Omega$, resulting in a propagation angle of $\beta=$ $\pi / 4$. The inertial waves are axisymmetric and originate at the corners where the boundary conditions are discontinuous and the corner flows form. When the waves propagate towards the axis, their energy concentrates in a region around the cylinder center, where the inertial waves from both corners meet. This results in the formation of a toroidal region where the velocity and vorticity of the flow reach a large value, the largest in the bulk of the flow, although smaller that the corresponding values inside the (very thin) sidewall boundary layer.

It is of interest to determine how the thickness and intensity of the shear layer scales with $\Omega$. From the contours of $\eta$ for $\Omega \in\left[10^{4}, 10^{6}\right]$ shown in Fig. 3 it is apparent that the shear layers vary self-similarly with $\Omega$. To quantify this, we use the maximum of $\eta$ at the midheight near the axis $\eta_{\max }$ (which corresponds to the maximum in the toroidal vortex) and its radial location $r_{\max }$. Figure 5(a) shows radial profiles of $\eta$ near the axis at midheight from which $\eta_{\max }$ and $r_{\max }$ are determined. Figures 6(a) and 6(b) show log-log plots of $\eta_{\max }$ vs $\Omega$ and of $r_{\max }$ vs $\Omega$, from which we have

$$
\eta_{\max } \sim \Omega^{1 / 3} \text { and } r_{\max } \sim \Omega^{-1 / 3} \text {. }
$$

Figure 6(c) shows that $w_{\max }$ has a small variation (less than $15 \%$ ) over the three decades of variation in $\Omega$ shown, with the variation at the higher frequencies being much smaller. This small variation suggest that the velocity field scales with the amplitude of the forcing, that has been kept constant $\left(\operatorname{Re}=10^{2}\right)$ for the results shown in the figure, and is almost independent of the frequency $\Omega$. This explains the vorticity scaling as the ratio of the velocity scaling (constant) divided by the length scaling of the inertial waves $\Omega^{-1 / 3}$. It is also interesting to determine the decay rate of the inertial wave beams as they propagate. According to [19], inertial waves decay exponentially with the spin-up time, which in terms of our nondimensional variables is given by $\Omega^{-1 / 2}$. If $t_{1}$ is the time taken by the inertial wave to travel a typical length $L$ (we will take $L$ to be the radius of nondimensional length one), we then expect a decay of the form $\exp \left(-\alpha \Omega^{1 / 2} t_{1}\right)$, where $\alpha$ is some $O(1)$ constant. We can estimate $t_{1}=L / c_{g}$, where $c_{g}$ is the group velocity of the inertial wave, given by $c_{g}=(2 \Omega / k) \sin \beta$ [3], where $\beta$ is the angle in the dispersion relation (9). We also need an estimate of the wave-vector modulus $k=2 \pi / \lambda$, where $\lambda$ is the wavelength. From Fig. 4, we see that any geometric length of the inertial wave scales as $r_{\max }$, so $\lambda \propto \Omega^{-1 / 3}$. Putting everything together, we arrive at an estimate for the attenuation rate of the inertial waves:

$$
\psi=\psi_{0} \exp \left[-\alpha \Omega^{-1 / 6}(\sin \beta)^{-1}\right],
$$

where the various constants of proportionality have been included in $\alpha . \psi$ is any velocity (or vorticity) component of the flow along the inertial wave beam, at unit distance from the beam source, where the value of $\psi$ is $\psi_{0}$. The $(\sin \beta)^{-1}$ comes from the group velocity dependence on $\sin \beta$. The variation of the decay rate with $\Omega$ is slow, due to the exponent $-1 / 6$. We observe that with increasing $\Omega$ the attenuation of the wave decreases; therefore, there is a critical value of $\Omega$ above which the attenuation is small enough so that the inertial waves are well established throughout the whole domain. From Fig. 3, we observe that this value of $\Omega$ is about $10^{3}$.

The results presented so far are all for a fixed sidewall oscillation amplitude of $\operatorname{Re}=10^{2}$. Now we explore what happens when varying Re. Figure 7 shows the radial profiles of (a)

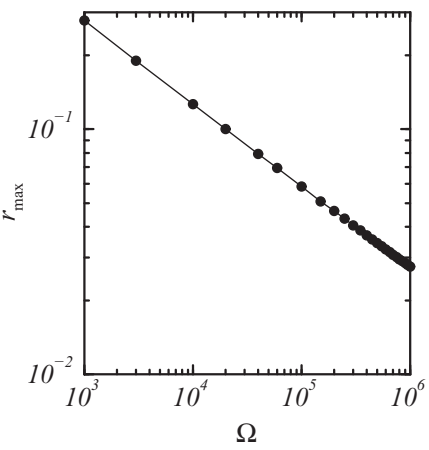

(b)

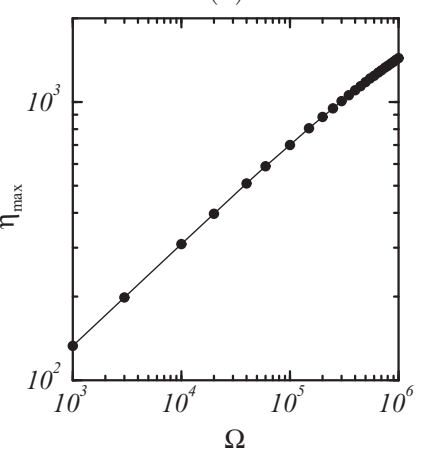

(c)

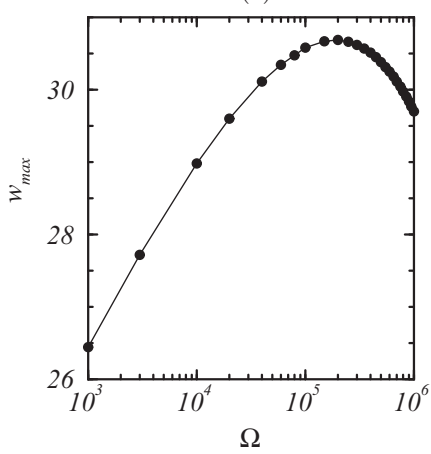

FIG. 6. Variation with $\Omega$ of the maximum $w$ and $\eta$ in $(z=0, r \in[0,0.5])$ for $\operatorname{Re}=10^{2}, \Gamma=2$, and $F=0.7071068$, together with the radial location where the maximum occurs. 


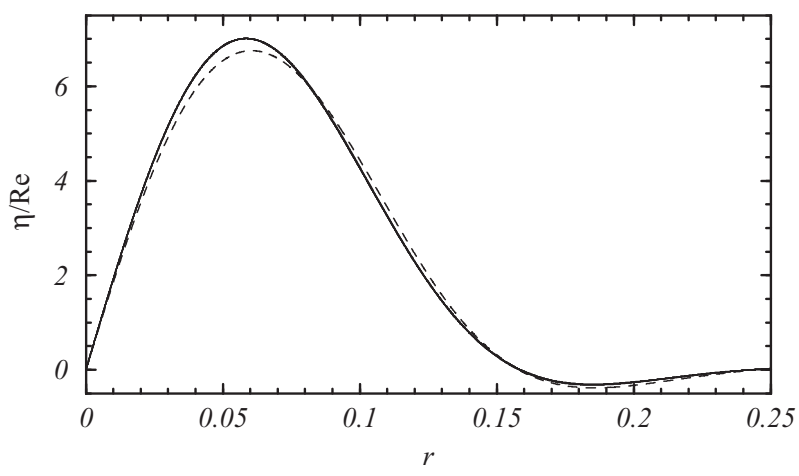

FIG. 7. Radial profiles of $\eta$, scaled by Re, at $z=0$ near the axis, for $\Omega=10^{5}, \Gamma=2, F=0.7071068$, and $\operatorname{Re}=1,30,100,300,10^{3}$, $3 \times 10^{3}$, and $10^{4}$, showing linear scaling of $\eta$ with Re except for the $\operatorname{Re}=10^{4}$ case (dashed curve). All cases used $n_{r} \times n_{z}=96 \times 128$ and $\delta t=T / 5000$.

$\eta$ scaled by $\mathrm{Re}$, at midheight, for a wide range of $\operatorname{Re} \in\left[1,10^{4}\right]$ with fixed $\Omega=10^{5}, \Gamma=2$, and $F=0.7071068$. We see that all the profiles, except for the largest $\mathrm{Re}=10^{4}$ case, collapse with the linear Re scaling. So, we have

$$
\eta_{\max } \sim \operatorname{Re} \Omega^{1 / 3} \text { and } r_{\max } \sim \Omega^{-1 / 3} .
$$

These results are consistent with the asymptotic analysis of [43] which gives that the thickness of the shear layers, for forcing frequencies that are not too small, scale with $\Omega^{-1 / 3}$.As the vorticity is the space derivative of the velocity, $\eta=\partial_{z} u-$ $\partial_{r} w$, and the scale of the spatial variation is set by $r_{\max }$, we conclude that the scaling of the velocity is independent of $\Omega$,

$$
w=O\left(\eta_{\max } r_{\max }\right)=O\left(\operatorname{Re} \Omega^{1 / 3} \Omega^{-1 / 3}\right)=O(\operatorname{Re}),
$$

in good agreement with the scaling of the velocity of the oscillating Stokes boundary layer [44,45].

The nonlinearities at the largest amplitude lead to the departure from linear scaling. Figure 8 shows the $\eta$ contours at $\operatorname{Re}=1$ and $\operatorname{Re}=10^{4}$. We see that the structure of $\eta / \operatorname{Re}$ is identical in the two cases except in the neighborhood of the corners where there is a slight formation of a stronger corner vortex at the top (bottom) corner when the sidewall moves down (up) that breaks the $K$ symmetry, as is to be expected since increasing the forcing amplitude Re leads to larger values of the nonlinear advection terms in the Navier-Stokes equations, and these break the $K$ symmetry.

\section{A. Linear stability analysis}

So far, we have discussed the properties of the inertial waves in a confined fluid system, assuming that the flow is axisymmetric. The stability of the computed flows to three-dimensional perturbations must therefore be analyzed in order to determine if these flows are stable and observable in experiments. The oscillating Stokes boundary layer is very robust [46], and this was one of the main reasons for choosing the present setting. The linear stability analysis is performed via time evolution of the Navier-Stokes equations. The stability of the periodic axisymmetric basic state is determined by introducing small random perturbations into all azimuthal Fourier modes. For sufficiently small perturbations, the non- (a) $\operatorname{Re}=1.0$

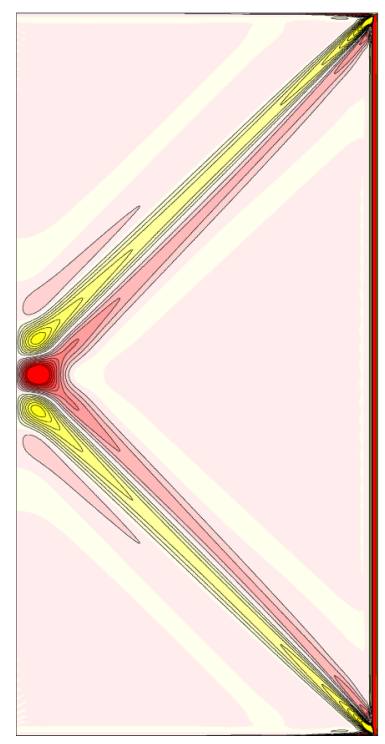

FIG. 8. (Color online) Contours of the azimuthal vorticity for $\Omega=10^{5}, \Gamma=2, F=0.7071068$, and $\mathrm{Re}$ as indicated, at a phase where the displacement of the sidewall is maximum in the negative- $z$ direction. There are 10 contour levels in the ranges $\eta \in[-5,5]$ for $\mathrm{Re}=1.0$ and $\eta \in\left[-5 \times 10^{4}, 5 \times 10^{4}\right]$ for $\mathrm{Re}=10^{4} ; n_{r} \times n_{z}=$ $96 \times 128$ and $\delta t=T / 5000$.

linear couplings between Fourier modes are below round-off numerical noise and the growth rates and eigenfunctions corresponding to the fastest growing perturbations at each Fourier mode emerge from time evolution. This is analogous to a matrix-free generalized power method for steady basic states $[47,48]$, generalized to periodic basic states. Figure 9(a) shows an example of the time evolution of the modal kinetic energies associated with three-dimensional perturbations for a moderate forcing amplitude $\operatorname{Re}=10^{2}$ with $\Omega=10^{3}, \Gamma=2$, and $F=0.7071068$. The modal kinetic energy of the Fourier modes corresponding to azimuthal wave numbers $m$ are

$$
E_{m}=0.5 \int_{-0.5 \Gamma}^{0.5 \Gamma} \int_{0}^{1} \boldsymbol{u}_{m} \cdot \boldsymbol{u}_{m}^{*} r d r d z,
$$

where $\boldsymbol{u}_{m}$ is the $m$ th Fourier mode of the velocity field and $\boldsymbol{u}_{m}^{*}$ is its complex conjugate. The energy of the axisymmetric state in this case is $E_{0} \approx 5.5 \times 10^{4}$. The initial perturbation energies, $E_{m}$ with $m>0$, introduced at $t=0$, are $10^{18}$ times smaller than $E_{0}$ and they decay, without any nonlinear interactions between them, by about 14 orders of magnitude to machine zero in about 0.2 viscous time units. We have used 128 Fourier modes in the azimuthal direction, but only plotted the energies of the first 64 because the energies decay faster with increasing azimuthal wave number, as can be seen in Fig. 9(b). The least damped eigenvalue corresponds to $\mu_{1}=-58.58$. It is clear from these results that the considered flow is stable.

If we increase the sidewall oscillation amplitude $\mathrm{Re}$, the flow eventually becomes unstable. For the case considered in Fig. 9, but with $\operatorname{Re}=10^{3}$, the flow is still stable. However, for $\operatorname{Re}=2 \times 10^{3}$ the axisymmetric flow has undergone a symmetry-breaking bifurcation to a modulated rotating wave state, illustrated in Fig. 10 and the associated 
(a)

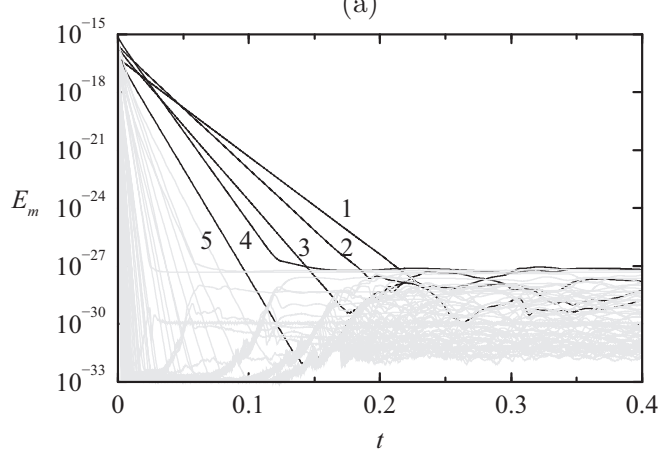

(b)

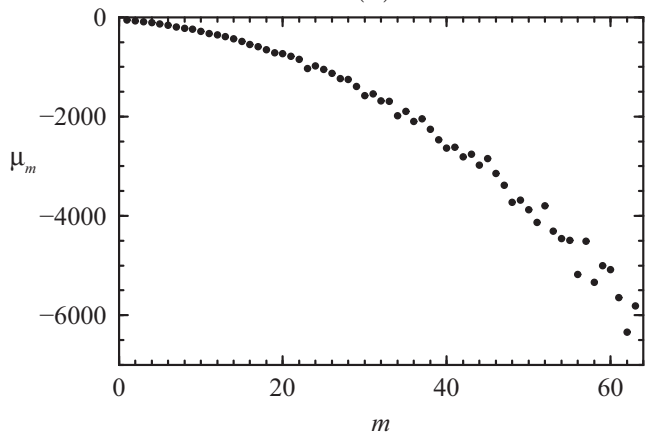

FIG. 9. Decay of the nonzero Fourier modes for the axisymmetric solution at $\Omega=10^{3}, \Gamma=2, \operatorname{Re}=10^{2}$, and $F=0.7071068$; 64 Fourier modes displayed. (a) Time series of the energies $E_{m}$; (b) least damped eigenvalue $\mu_{m}$ for each mode $m$.

online movie [42]. This figure shows isosurfaces of the nonaxisymmetric contribution to the azimuthal vorticity $\eta$, at ten equispaced phases over one forcing period. It is evident that the instability mechanism acts near the corners, where the axial oscillations of the sidewall produce alternate rollers in each corner during one forcing period. On these rollers, braids are formed with an azimuthal wave number $m=12$. The instability mechanism is not associated with the inertial wave beams. They are an instability of the rollers that form due to the axial oscillations of the sidewall. The instability depends critically on the amplitude of these oscillations, Re. Physically, these rollers are fed by the volume of fluid in the sidewall layer and, when the volume of fluid injected into the rollers is sufficiently large, they become unstable. This volume of fluid depends on the total displacement of the sidewall during each oscillation half-cycle, measured by $\delta h / a=\operatorname{Re} / \Omega_{f}$. In the case under consideration, $\Omega_{f} \approx 1414$, and the instability sets in between $\operatorname{Re}=10^{3}(\delta h / a \approx 0.707)$ and $\operatorname{Re}=2 \times 10^{3}$ $(\delta h / a \approx 1.414)$, suggesting that the rollers become unstable when $\delta h / a \approx 1$, that is, when the sidewall displacement during each half-cycle is comparable to the cylinder radius. The inertial waves continue to exist in the bulk of the flow, but are now distorted by the three-dimensional instability of the rollers, as shown in Fig. 11. The contours of $\eta$ have been quadratically spaced in order to show the inertial waves, that are much weaker than the rollers; compare with Fig. 3(c) corresponding to the axisymmetric solution at a much lower $\operatorname{Re}=10^{2}$.

Figure 12 shows the power spectral density of the axial velocity at a point near the center of the sidewall, showing that the bifurcated state is quasiperiodic with two (a) 0

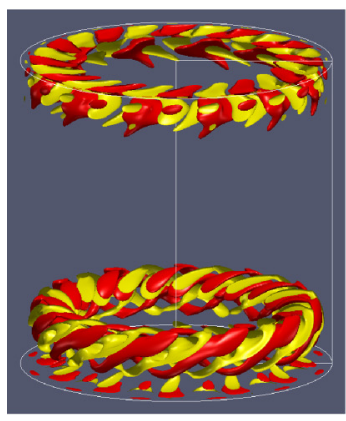

(f) $0.5 \tau$

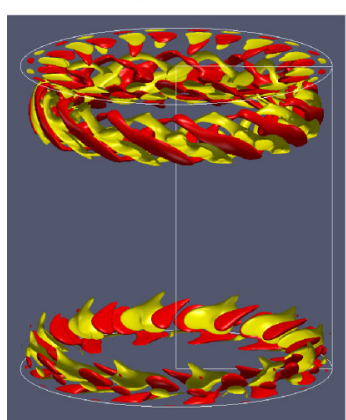

(b) $0.1 \tau$

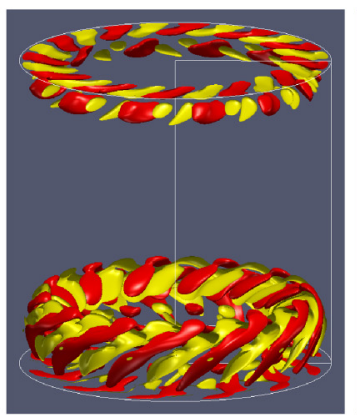

(g) $0.6 \tau$

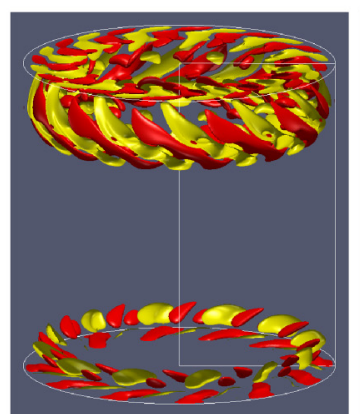

(c) $0.2 \tau$

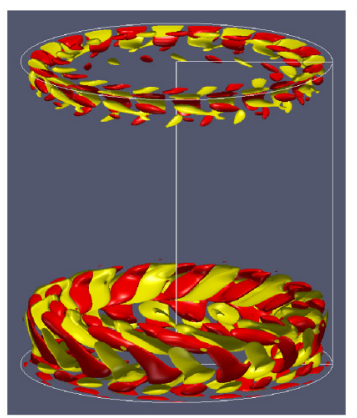

(h) $0.7 \tau$

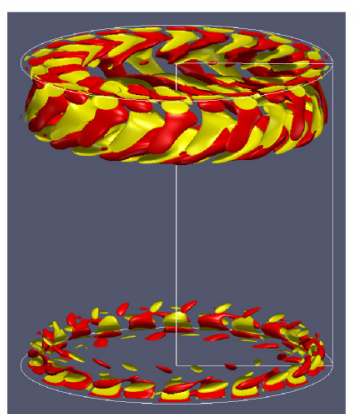

(d) $0.3 \tau$

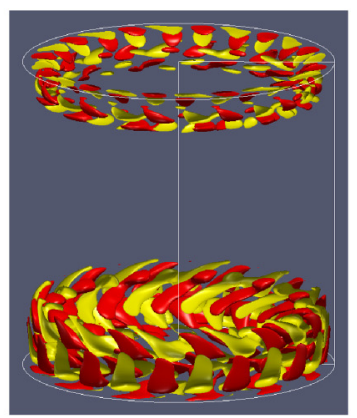

(i) $0.8 \tau$

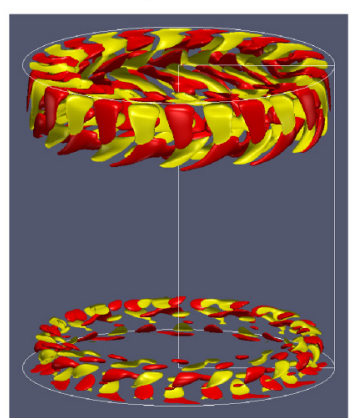

(e) $0.4 \tau$

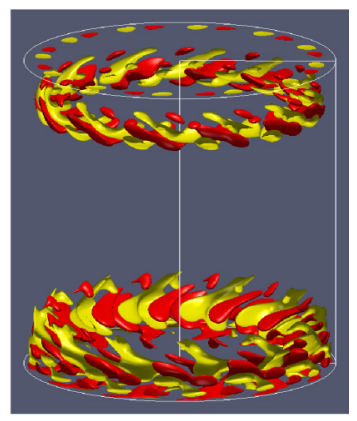

(j) $0.9 \tau$

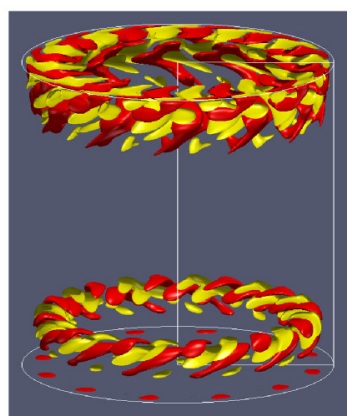

FIG. 10. (Color online) Isosurfaces of the nonaxisymmetric contributions to the azimuthal vorticity at levels $\eta_{3 \mathrm{D}}= \pm 1000$ for the modulated rotating wave state at $\Omega=10^{3}, \Gamma=2, \operatorname{Re}=2 \times 10^{3}$, and $F=0.7071068$ shown at 10 phases over one forcing period $\tau=2 \pi / \Omega_{f}=\pi /(F \Omega)$. See the associated online movie [42]. 
(a) 0

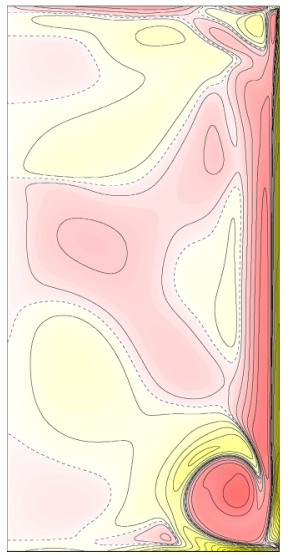

(b) $\tau / 6$

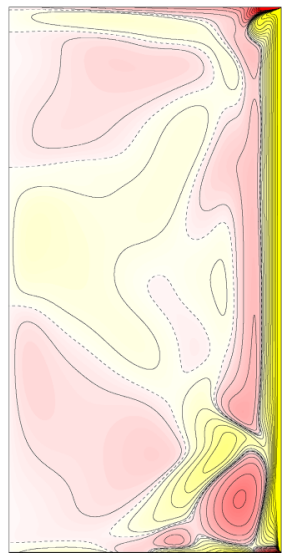

(c) $2 \tau / 6$

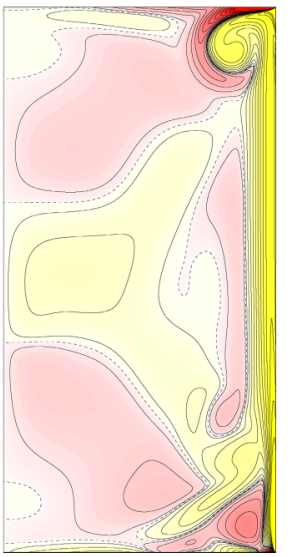

(d) $3 \tau / 6$

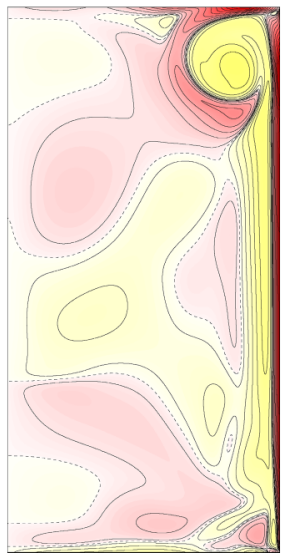

(e) $4 \tau / 6$

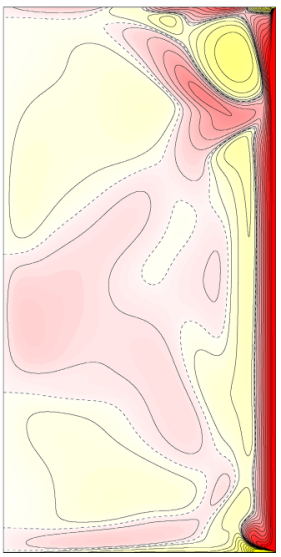

(f) $5 \tau / 6$

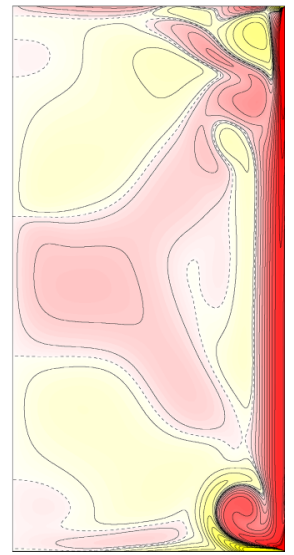

FIG. 11. (Color online) Contours of the azimuthal vorticity for the modulated rotating wave state at $\Omega=10^{3}, \Gamma=2, \operatorname{Re}=2 \times 10^{3}$, and $F=0.7071068$ shown at six phases over one forcing period $\tau=2 \pi / \Omega_{f}=\pi /(F \Omega)$. There are 24 quadratically spaced contour levels in the range $\eta \in\left[-5 \times 10^{4}, 5 \times 10^{4}\right]$. See the associated online movie [42].

frequencies, the forcing frequency $\Omega_{f}$, and another frequency $\Omega_{p}$ that corresponds to the precession of the modulated rotating wave, as is obvious by looking at the movie of the solution [42].

By increasing the rotation rate of the cylinder $\Omega$ while keeping Re fixed, the Stokes boundary layer becomes more stable. The rapid oscillations make it more difficult for instabilities to develop because the time between oscillations is smaller. We have verified this by computing the least damped eigenvalues of the axisymmetric base state for $\mathrm{Re}=100$ and different $\Omega$. The results presented in Fig. 13 show that the flow becomes more stable with increasing $\Omega$ for a fixed Re. All the axisymmetric solutions we have analyzed for $\mathrm{Re}=100$ are stable, confirming the previous considerations.

So, in summary, for rapid rotations ( $\Omega$ large) subjected to small amplitude forcing (Re small), which is the appropriate setting for the study of inertial waves, the system under consideration is extremely robust to perturbations and affords one the ability to study inertial waves in a physically realizable setting unencumbered by instabilities.

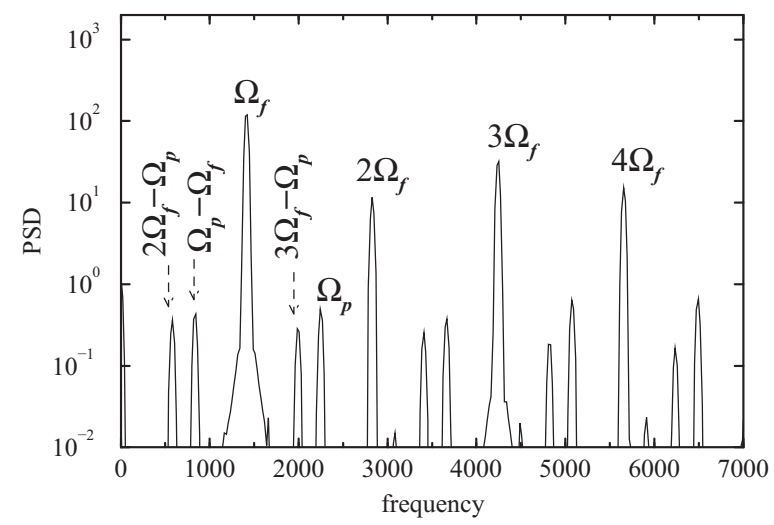

FIG. 12. Power spectral density of the axial velocity at the point $(r, \theta, z)=(0.9,0,0)$ over about 260 forcing periods of the modulated rotating wave state at $\Omega=10^{3}, \Gamma=2, \operatorname{Re}=2 \times 10^{3}$, and $F=0.7071068$.

\section{B. Comparing the nonlinear wave beams with inviscid modes and ray tracing}

In order to measure the dependence of the inertial wave beam intensity on the forcing frequency, i.e., the response diagram of our forced system, we have computed the maximum absolute value of the azimuthal vorticity, $\max |\eta|$, in the fluid domain. As $\eta$ is very large at the boundary layers, we have excluded them from the maxima computation. We have fixed $\Omega=10^{3}, \Gamma=2$, and $\operatorname{Re}=10^{2}$, and varied $F$ (i.e., the forcing frequency) over the range where inertial waves are present, $0.01 \leqslant F \leqslant 1$ in steps of 0.01 . The resulting values of $\max |\eta|$ as a function of $F$ are plotted in Fig. 14 as solid symbols, and a spline fit (the blue line) has been added to guide the eye. The most salient features of the figure are the large variations in $\max |\eta|$ with very small changes in $F$. On average, the values of $\max |\eta|$ closely follow the decay rate estimate given by (15). The best fit to this decay rate is shown in the figure as the thick orange monotonic line, given by

$$
\max |\eta|=A \exp \left[-\alpha \Omega^{-1 / 6}\left(1-F^{2}\right)^{-1 / 2}\right],
$$

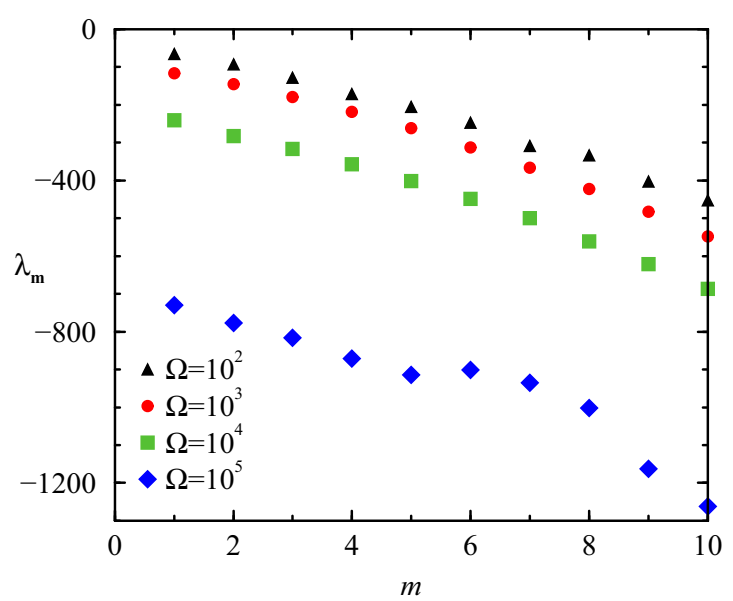

FIG. 13. (Color online) Least damped eigenvalues for the axisymmetric base state at $\Gamma=2, \operatorname{Re}=10^{2}$, and $F=0.7071068$, for different $\Omega$ values as indicated. 


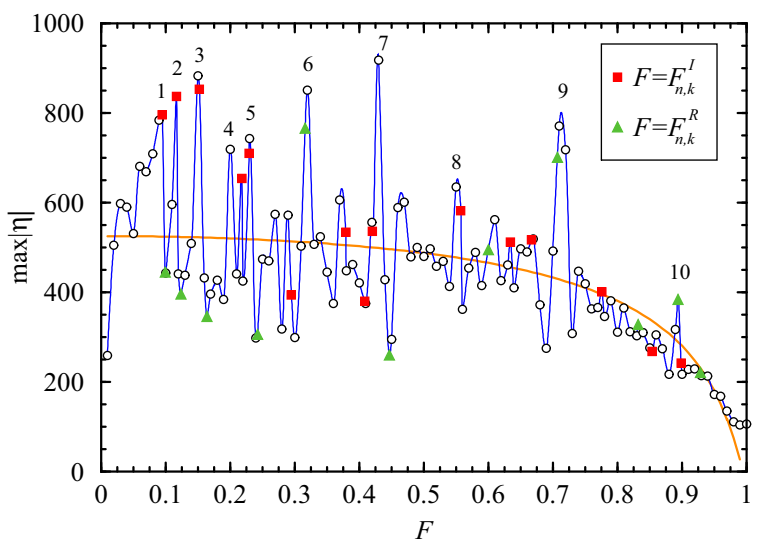

FIG. 14. (Color online) Response diagram: symbols are the maximum values of $|\eta|$ in the whole domain excluding the boundary layers, as a function of $F$, for $\Omega=10^{3}, \Gamma=2$, and $\operatorname{Re}=10^{2}$. The thin solid (blue) line is a spline fit to guide the eye. The thick gray (orange) monotonic line is the theoretical value of $|\eta|$ given by (19). The (red) squares correspond to the $F$ values of the inviscid Kelvin modes (IM) and the (green) triangles correspond to the $F$ values of the retracing rays (RR), for values $n+k \leqslant 6$. The precise values of the IM and RR cases are listed in Table I. The relevant peaks associated with IM and/or RR have been numbered for further reference. Contours of $\eta$ for all the computed cases in the diagram are included in the accompanying movie [42], where time is replaced by increasing $F$ values.

with $A=852.2$ and $\alpha=3.295$. This is an important result, that must however be completed with an explanation of the large variations in the inertial wave intensity when varying $F$. This will be accomplished by comparing these results with the inviscid eigenmodes of the cylindrical cavity, and also with ray-tracing techniques.

The eigenmodes of the cylindrical cavity are the solutions of the inviscid linearized Euler equations, satisfying the impenetrability boundary condition at the walls. They can be expressed in terms of radial Bessel functions and trigonometric functions in the axial and azimuthal coordinates $[1,49]$. The azimuthal vorticity $\eta$ of the axisymmetric eigenmodes is given by

$$
\begin{gathered}
\eta_{n, k}(r, z)=J_{1}\left(\delta_{k} r\right) \sin n \pi\left(\frac{z}{\Gamma}+\frac{1}{2}\right), \\
\tan \beta_{n, k}^{I}=\frac{\Gamma \delta_{k}}{n \pi}, \quad F_{n, k}^{I}=\cos \beta_{n, k},
\end{gathered}
$$

where $n, k$ are positive integers characterizing the different eigenmodes, $\delta_{k}$ is the $k$ zero of the Bessel function $J_{1}, \beta_{n, k}^{I}$ is the angle between the horizontal plane and the wave beam (9), and $F_{n, k}^{I}$ is the $F$ value associated to the inviscid $(n, k)$ eigenmode. The number of zeros of $\eta_{n, k}$ in the interior of the fluid domain, in the $r$ and $z$ directions, are $k-1$ and $n-1$, respectively. These functions are called the inviscid modes (IMs) or the Kelvin eigenmodes of the cylindrical cavity. Figure 15 shows some of these inviscid modes. Contours of the azimuthal vorticity are depicted for the IMs in the first row, while the second row shows the numerically obtained solution of the Navier-Stokes equations for the same values of the parameters. The solutions are very different for small values of $n(n \leqslant 3)$; however, they look increasingly similar with increasing $n$, differing only near the boundary layers; for $k>1$ and arbitrary $n$ they are very different. The differences are due mainly to the fact that the wave beams are generated at the corners, so the Navier-Stokes solutions always exhibit strong wave beams emerging from the corners, while the IMs do not have a well defined origin of the waves. However, when $F$ is very small, the angle $\beta$ is close to $\pi / 2$, and the wave beams are almost vertical; they emerge not from the corner but from the whole sidewall boundary layer, resulting in vertical strips that closely resemble the IM in the bulk. In general, one could expand the Navier-Stokes solutions in the bulk in terms of the IM [1]; however, a very large number of IMs will be necessary to reproduce viscous results, except when $F$ is very small. In fact, these expansions are not convergent due to the presence of the boundary layers, and care must be taken when using them [50].

In Fig. 14 we have included the Navier-Stokes solutions computed at the $F$ values of the inertial modes, $F_{n, k}^{I}$, for $n+$ $k \leqslant 6$. We clearly see that they are not associated with the large response peaks, and therefore the response peaks do not appear at the eigenmode frequencies, except for small values of the forcing frequency, $F \lesssim 0.2$. The small $F$ cases precisely coincide with the $(n, k)$ values for which the Navier-Stokes solutions and the inviscid modes are very similar.

In many rotating fluids the inertial wave beams originate in specific locations inside the fluid container. From these specific locations, beams of inertial waves emerge, at angles given by the dispersion relation (9). Therefore, much can be predicted by ray tracing the characteristics from the origin of the inertial waves and successively reflecting the rays on the container walls. Phenomena such as wave focusing and wave attractors have been analyzed using ray tracing [51-54]. In our problem, the wave beams originate at the corners where the end walls and the sidewall meet. These beams will reflect at domain boundaries and in general they never retrace themselves, densely filling the domain, except for particular values of the angle $\beta$. Due to the axisymmetry of the solutions for the parameter values analyzed here, when the beam crosses the cylinder axis it is the same as a reflection on the axis, and we only need to consider the domain $(r, z) \in[0,1] \times[-\Gamma / 2, \Gamma / 2]$ and reflections on its walls to trace the ray beam trajectories. A trajectory starting in one corner is closed if it arrives at any other corner after $n-1$ vertical and $k-1$ horizontal reflections, where $n$ and $k$ are positive integers characterizing the retracing ray (RR). The first row in Fig. 16 shows six RR for the specified values of $(n, k)$, where the RR emerging from both sidewall corners have been plotted. The angle and $F$ value for the retracing ray $(n, k)$ are given by

$$
\tan \beta_{n, k}^{R}=\frac{\Gamma k}{n}, \quad F_{n, k}^{R}=\cos \beta_{n, k}=\frac{n}{\sqrt{n^{2}+\Gamma^{2} k^{2}}} .
$$

The second row in Fig. 16 shows the Navier-Stokes solutions corresponding to the frequencies $F_{n, k}^{R}$ of the RR in row one. The ray structure of the Navier-Stokes solutions coincides with the corresponding retracing ray. However, the rays are no longer characteristic lines of zero width, but they have a finite width due to viscous effects that smooth out the discontinuities of the characteristics of the inviscid linearized hyperbolic problem. We could expect that the viscous solutions corresponding to $F_{n, k}^{R}$ would correspond to the peaks in the response diagram, however, this is not exactly the case. What we observe is that there is always a peak response very close to the $F_{n, k}^{R}$ values, 

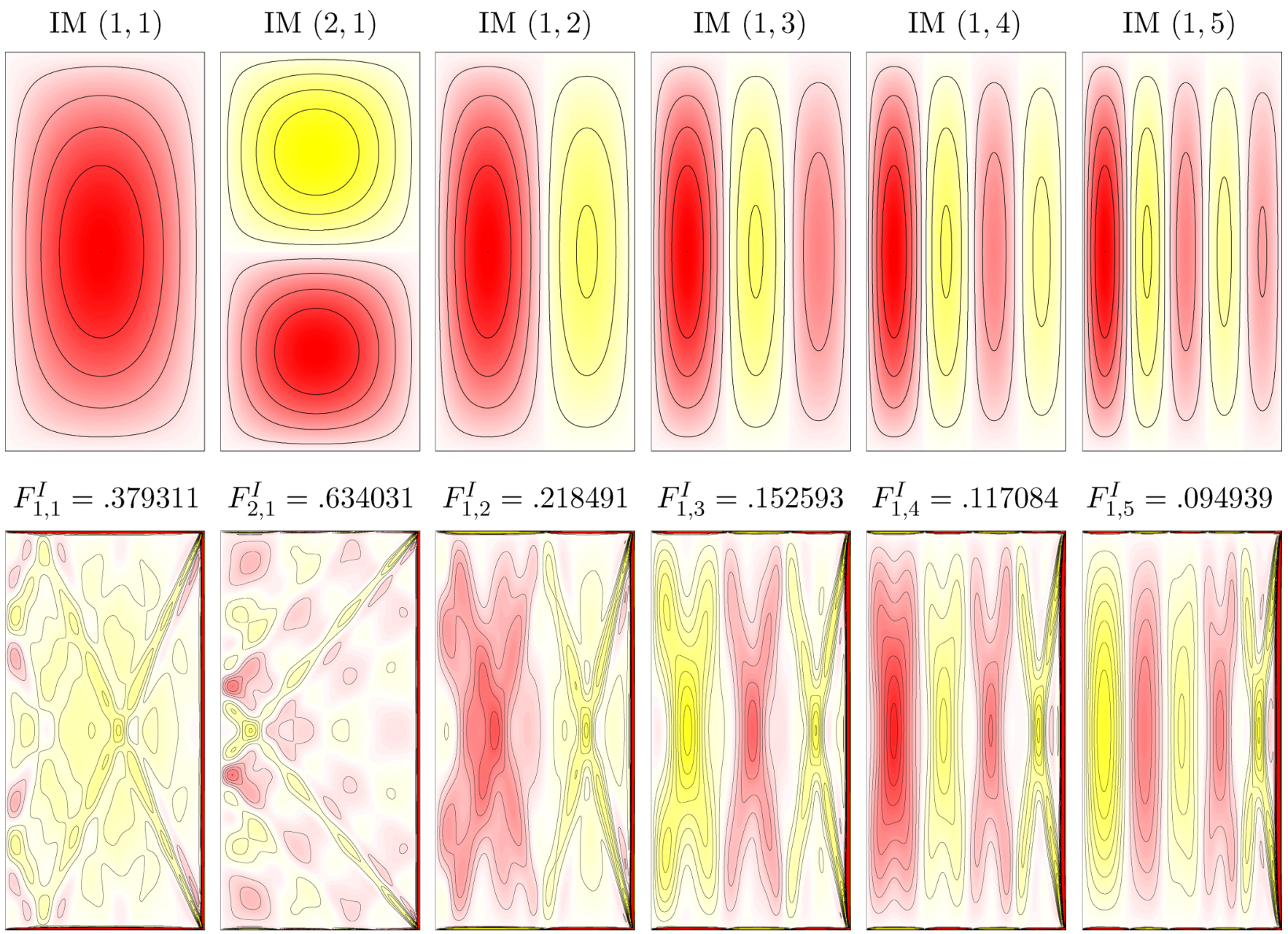

FIG. 15. (Color online) Comparison between the Kelvin eigenmodes, also called inviscid modes IM (first row), and the Navier-Stokes solution at the same $F$ value as indicated. The eigenmodes are labeled by $(n, k)$.

with the same wave beam structure, as shown in the third row in Fig. 16. The contour levels and color map (gray scale) in the two last rows are the same in all figures, corresponding to $\eta \in[-1000,1000]$, so a stronger color (gray scale) intensity corresponds to a larger peak in the response diagram.

The small discrepancy in $F$ between the retracing rays and the Navier-Stokes solutions at the maximums of the response diagram is in part due to the presence of the boundary layers. The inertial wave beams do not originate exactly at the corner, but close by where the sidewall and end wall boundary layers meet. Moreover, the wave beams are not reflected at the walls, but at the boundary layers that are very narrow but not of zero thickness. Furthermore, Borcia and Harlander [53] have shown that when curvature and viscosity are neglected, the ray pattern gives the exact eigenmode frequency, and so another source of discrepancy between the rays and the Navier-Stokes solutions come from the effects of curvature and viscosity. Another important feature of these solutions is the strong interaction between the inertial wave beams and the boundary layers. This is clearly observed at the end wall boundary layers that become thicker when interacting with the wave beam. Finally, we observe that the small differences in $F$ between the solutions in the top and bottom rows of Fig. 16 result in significant variations of the beam intensities.
It is apparent that the thickness of the shear layers and how they dissipate depends on $F$. This is not too surprising given that the Stokes layer that drives the shear layers has a thickness which scales with $F^{-1 / 2}$ for fixed $\Omega$, and also the structure of the corner flow is affected by the angle at which the shear layer emerges from the corner, and this angle is completely determined by $F$. Moreover, Fig. 14 shows that the attenuation of the shear layers is strongest for the smallest angle of propagation $\beta$ (i.e., $F$ closest to one). This agrees very well with the decay rate estimate (15) for the inertial waves. Another important feature of these shear layers, which was already evident for the $F=1 / \sqrt{2}$ cases studied in detail above, is how the shear layer thickens at smaller radii. This is a purely geometric effect: the inertial waves are axisymmetric and originate at the corners where the sidewall boundary layer forms a corner jet due to the axial oscillations of the sidewall. The energy injected in this way becomes concentrated in a smaller volume as the wave beam approaches the cylinder axis. Finally, the superposition of the different beams emerging from the top and bottom corner and their reflections off the cylinder walls may result in constructive or destructive interference. The result depends critically on the geometry of the container and on the precise value of $F$, explaining the large variations in the intensity of the wave beams with small variations of $F$. This 
$\mathrm{RR}(1,1)$

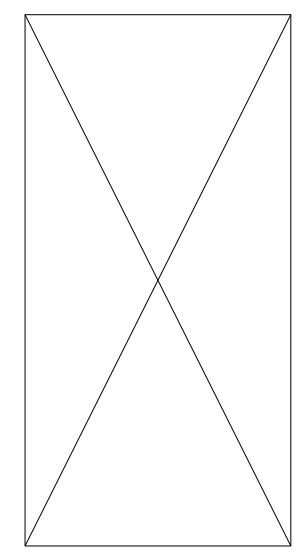

$F_{1,1}^{R}=0.4472$

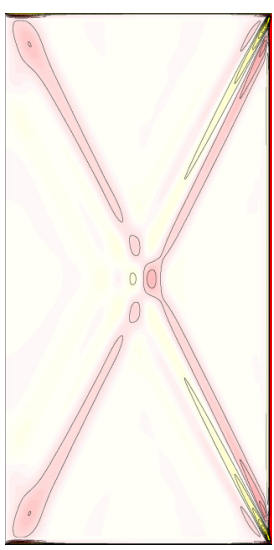

$F=0.43$

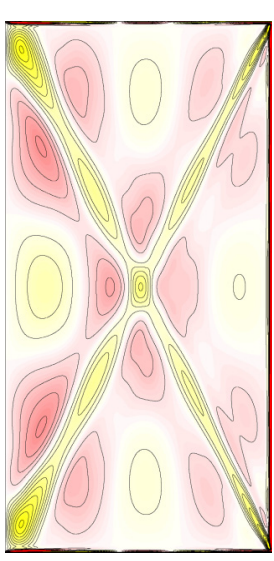

$\mathrm{RR}(2,1)$

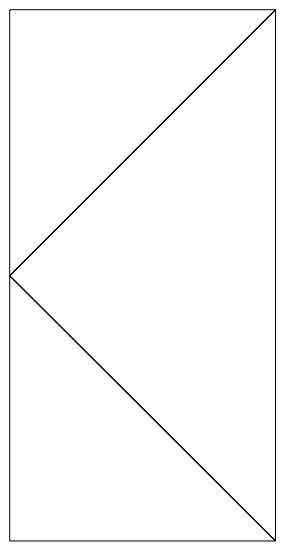

$F_{2,1}^{R}=0.7071$

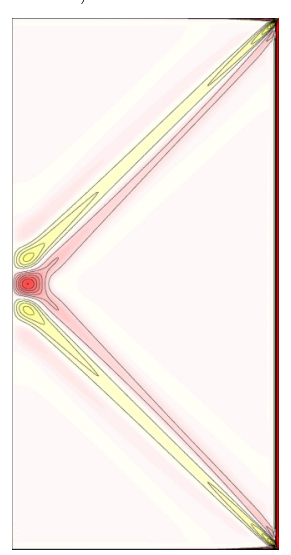

$F=0.71$

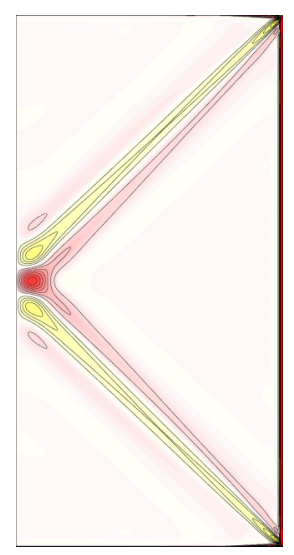

$\mathrm{RR}(1,2)$

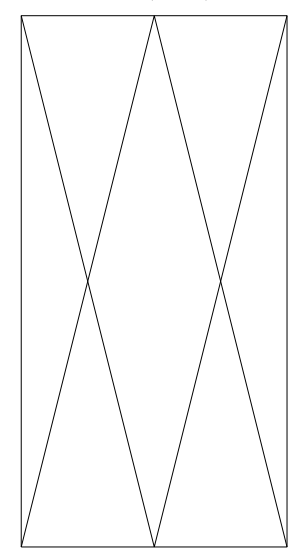

$F_{1,2}^{R}=0.2425$

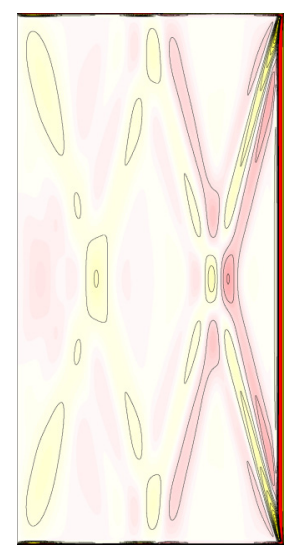

$F=0.23$

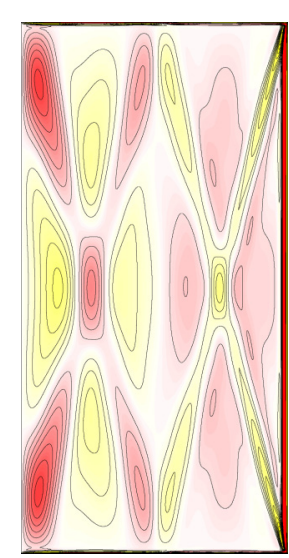

$\mathrm{RR}(1,3)$

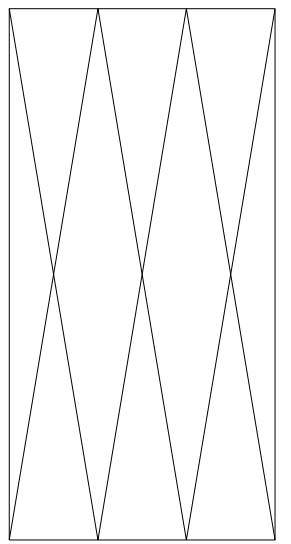

$F_{1,3}^{R}=0.1644$

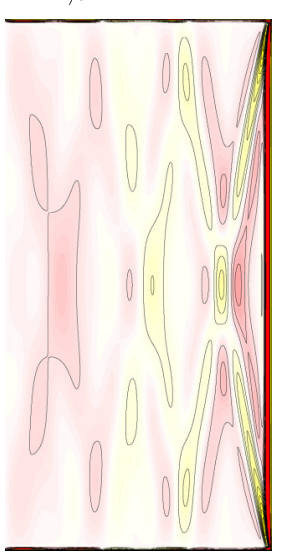

$F=0.15$

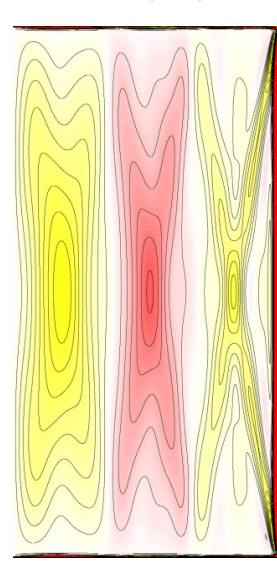

$\mathrm{RR}(2,3)$

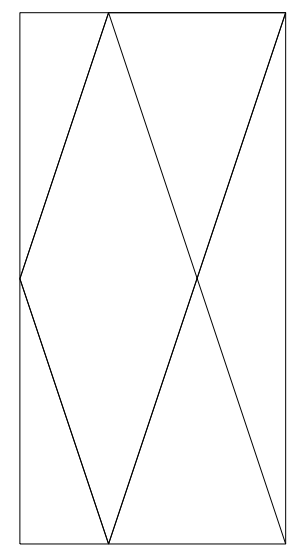

$F_{2,3}^{R}=0.3162$

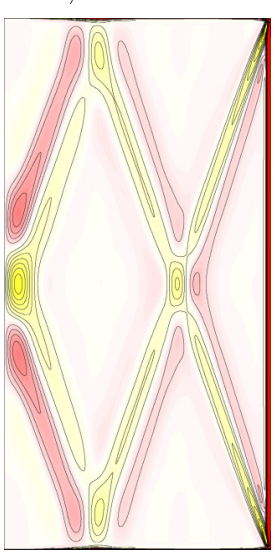

$F=0.32$

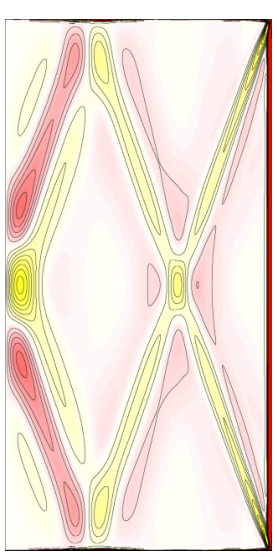

$\operatorname{RR}(1,5)$

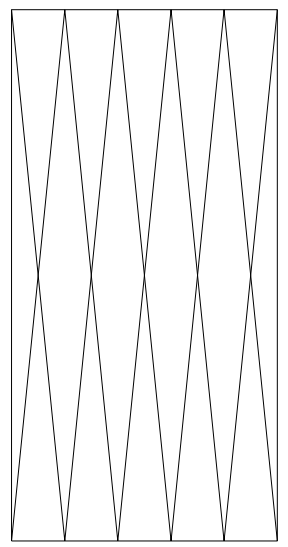

$F_{1,5}^{R}=0.0995$

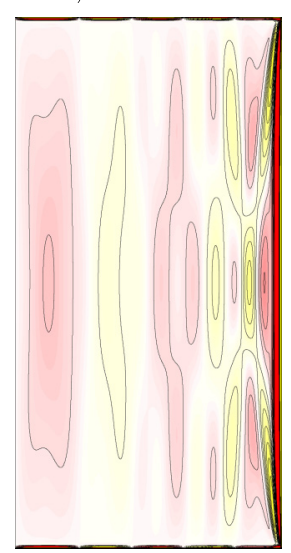

$F=0.094939$

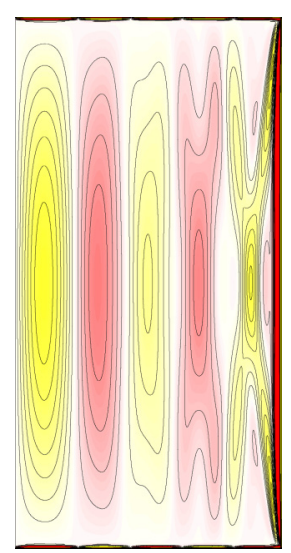

FIG. 16. (Color online) Top row shows the characteristic rays at $F$ corresponding to the indicated retracing rays. The middle row shows $\eta$ contours at $\Omega=10^{5}, \operatorname{Re}=10^{2}, \Gamma=2$, and $F$ at the same values corresponding to the indicated retracing rays, at a phase where the displacement of the sidewall is maximum in the negative- $z$ direction. The bottom row shows $\eta$ contours for the same parameters as in the middle row, but for slightly different values of $F$ as indicated. For all contour plots, there are 10 contour levels in the range $\eta \in[-1000,1000]$.

is clearly observed in the movie [42] accompanying Fig. 14 that shows all the cases we have computed as a function of $F$.

An interesting question is the following: what is the relationship between the retracing rays solutions and the inertial modes? To address this, the first thing to do is compare the $F$ values corresponding to the inertial modes (20) and the retracing rays (21). When $k$ is large, the $k$ zero of the Bessel function of order one, $J_{1}\left(\delta_{k}\right)=0$, admits the asymptotic expansion [55]

$$
\begin{aligned}
\delta_{k} & =\left(k+\frac{1}{4}\right) \pi+O\left(\frac{1}{k}\right), \\
\tan \beta_{n, k}^{I} & =\Gamma\left(k+\frac{1}{4}\right) \frac{1}{n}+O\left(\frac{1}{n k}\right) \gtrsim \tan \beta_{n, k}^{R}, \\
F_{n, k}^{I} & \lesssim F_{n, k}^{R} .
\end{aligned}
$$


TABLE I. Frequency values $F$ and maximum azimuthal vorticity $\max |\eta|$ for the Navier-Stokes solutions computed at the $F$ values corresponding to the inviscid modes (IM) and retracing rays (RR). Also included are the relevant peaks in Fig. 14, indicated as max $n$.

\begin{tabular}{|c|c|c|}
\hline$F$ & $\max |\eta|$ & Comment \\
\hline 0.09494 & 796.20 & $\max 1, \mathrm{IM}_{1,5}$ \\
\hline 0.09951 & 444.84 & $\mathrm{RR}_{1,5}$ \\
\hline 0.11708 & 836.82 & $\max 2, \mathrm{IM}_{1,4}$ \\
\hline 0.12403 & 396.13 & $\mathrm{RR}_{1,4}$ \\
\hline 0.15000 & 882.46 & $\max 3$ \\
\hline 0.15259 & 853.33 & $\mathrm{IM}_{1,3}$ \\
\hline 0.16440 & 345.79 & $\mathrm{RR}_{1,3}$ \\
\hline 0.20000 & 719.29 & $\max 4$ \\
\hline 0.21849 & 653.81 & $\mathrm{IM}_{1,2}$ \\
\hline 0.22950 & 709.73 & $\mathrm{IM}_{2,4}$ \\
\hline 0.23000 & 743.30 & $\max 5$ \\
\hline 0.24254 & 306.15 & $\mathrm{RR}_{1,2}$ and $\mathrm{RR}_{2,4}$ \\
\hline 0.29505 & 394.04 & $\mathrm{IM}_{2,3}$ \\
\hline 0.31623 & 765.71 & $\mathrm{RR}_{2,3}$ \\
\hline 0.32000 & 850.99 & $\max 6$ \\
\hline 0.37931 & 533.64 & $\mathrm{IM}_{1,1}$ \\
\hline 0.40870 & 379.97 & $\mathrm{IM}_{2,2}$ \\
\hline 0.42030 & 536.14 & $\mathrm{IM}_{3,3}$ \\
\hline 0.43000 & 917.68 & $\max 7$ \\
\hline 0.44721 & 259.92 & $\mathrm{RR}_{1,1}$ and $\mathrm{RR}_{k, k}$ \\
\hline 0.55000 & 635.24 & $\max 8$ \\
\hline 0.55759 & 581.85 & $\mathrm{IM}_{3,2}$ \\
\hline 0.60000 & 495.34 & $\mathrm{RR}_{3,2}$ \\
\hline 0.63403 & 511.91 & $\mathrm{IM}_{2,1}$ \\
\hline 0.66715 & 516.85 & $\mathrm{IM}_{4,2}$ \\
\hline 0.70711 & 700.98 & $\mathrm{RR}_{2,1}$ and $\mathrm{RR}_{4,2}$ \\
\hline 0.71000 & 771.18 & $\max 9$ \\
\hline 0.77588 & 401.40 & $\mathrm{IM}_{3,1}$ \\
\hline 0.83205 & 328.03 & $\mathrm{RR}_{3,1}$ \\
\hline 0.85377 & 267.90 & $\mathrm{IM}_{4,1}$ \\
\hline 0.89443 & 384.47 & $\max 10$ and $R R_{4,1}$ \\
\hline 0.89875 & 241.86 & $\mathrm{IM}_{5,1}$ \\
\hline 0.92848 & 220.78 & $\mathrm{RR}_{5,1}$ \\
\hline
\end{tabular}

Therefore, for large $k$ values, the retracing rays corresponding to $(n, k)$ have an $F$ value very close to, but slightly larger than, the $F$ value of the inertial mode with the same $(n, k)$ values. Furthermore, we have also seen that the RR are close to a maximum of the response curve for all $(n, k)$ values. Table I lists $F$ and $\max |\eta|$ for all the relevant peaks in Fig. 14, and also the values corresponding to inertial modes $\mathrm{IM}_{n, k}$ and retracing rays $\mathrm{RR}_{n, k}$, for $n+k \leqslant 6$. The table shows the aforementioned relationship between the Navier-Stokes solutions at the peak response and the inertial modes and retracing rays.

\section{CONCLUSION}

A primary aim of this paper is to study inertial waves in as simple a setting as possible within an enclosure with no-slip (physical) boundary conditions. Traditionally, inertial waves have been studied from a hyperbolic system resulting from a singular perturbation and linearization of the Navier-
Stokes equations. The singular perturbation corresponds to neglecting the viscous terms, and hence the full set of boundary conditions cannot be imposed and generally the solutions of the singularly perturbed problem do not satisfy the boundary conditions of the full problem. Nevertheless, the hyperbolic problem provides significant insights into many aspects of the problem. Several physical experiments as well as theoretical asymptotic analysis and numerical simulations have tried to solve the system for large $\Omega$ to verify whether the solutions in the limit of large $\Omega$ and the eigenmodes and the ray tracing of the characteristics problem agree. One difficulty has been that the manner in which the inertial waves have been produced leads to flow instabilities as $\Omega$ is increased. In some cases it is clear that the instabilities are associated with the driving mechanisms (e.g., boundary layer instabilities), but it is not so clear in other examples. What we have attempted to do in this paper, successfully, is to design a physically realizable system in which inertial waves are generated for large $\Omega$ with a driving mechanism that is robust to perturbations.

We have shown via numerical linear stability analysis that three-dimensional instabilities set in when $\operatorname{Re} / \Omega \sim O(1)$ and this corresponds to periodic forcing with axial displacements of the sidewall of the order of the cylinder radius. This is a huge displacement which, on the one hand, is impractical to implement in a physical experiment, and, on the other hand, is of no interest from the perspective of studying inertial waves which are driven in rapidly rotating flows (large $\Omega$ ) by very small amplitude disturbances (small Re), so that the regime of interest is $\operatorname{Re} / \Omega \ll 1$. Furthermore, since the inertial wave beams are best visualized in terms of their azimuthal vorticity, $\eta$, and $\eta$ scales linearly with Re, for large $\Omega$ one can drive the inertial waves at a reasonable value of Re so that $\eta$ can be readily measured using, for example, a strobed particle image velocimetry (PIV) technique while still having $\operatorname{Re} / \Omega$ small and avoiding any flow instabilities. A particular advantage of the axial oscillations of the sidewall forcing is the prospect of studying nonlinear aspects of inertial waves with large amplitude, both numerically and experimentally.

An important consequence of the analysis presented in this study is that inviscid modes and retracing rays, although they are useful techniques that in some cases give a good qualitative description of the flow, cannot account for quantitative results like the precise frequency values where the response to the forcing reaches the maximum intensity, the value of these maxima intensities, and the details of the inertial beams and the associated interference pattern. Only carefully conducted experiments and direct numerical simulations of the Navier-Stokes solutions can provide an accurate picture of the structure of the inertial waves.

\section{ACKNOWLEDGMENTS}

This work was supported in part by the US National Science Foundation Grants No. DMS-0922864 and No. CBET1336410, and the Spanish Ministry of Education and Science Grant No. FIS2009-08821. 
[1] H. P. Greenspan, The Theory of Rotating Fluids (Cambridge University Press, Cambridge, UK, 1968).

[2] J. Lighthill, Waves in Fluids (Cambridge University Press, Cambridge, UK, 1978).

[3] L. D. Landau and E. M. Lifshitz, Fluid Mechanics, 2nd ed. (Pergamon Press, New York, 1984).

[4] J. Pedlosky, Geophysical Fluid Dynamics, 2nd ed. (Springer, New York, 1987).

[5] K. D. Aldridge and L. I. Lumb, Nature (London) 325, 421 (1987).

[6] J. Noir, D. Brito, K. Aldridge, and P. Cardin, Geophys. Res. Lett. 28, 3785 (2001).

[7] M. A. Calkins, J. Noir, J. D. Eldredge, and J. M. Aurnou, Phys. Fluids 22, 086602 (2010).

[8] J. Noir, F. Hemmerlin, J. Wicht, S. M. Baca, and J. M. Aurnou, Phys. Earth Planet. Inter. 173, 141 (2009).

[9] W. V. R. Malkus, Science 160, 259 (1968).

[10] P. Olson, J. Geophys. Res. 86, 10875 (1981).

[11] R. Hide, Philos. Trans. R. Soc. London A 306, 232 (1982).

[12] A. Tilgner, Phys. Fluids 17, 034104 (2005).

[13] D. H. Kelley, S. A. Triana, D. S. Zimmerman, A. Tilgner, and D. P. Lathrop, Geophys. Astrophys. Fluid Dyn. 101, 469 (2007).

[14] K. Stewartson, J. Fluid Mech. 5, 577 (1959).

[15] A. D. McEwan, J. Fluid Mech. 40, 603 (1970).

[16] R. Manasseh, J. Fluid Mech. 315, 151 (1996).

[17] Lord Kelvin, Philos. Mag. 10, 155 (1880).

[18] J. P. Vanyo, Rotating Fluids in Engineering and Science (Butterworth-Heinemann, Boston, 1993); (republished: Dover, New York, 2001).

[19] H. P. Greenspan, J. Fluid Mech. 22, 449 (1965).

[20] W. W. Wood, Proc. R. Soc. London A 293, 181 (1966).

[21] R. R. Kerswell and C. F. Barenghi, J. Fluid Mech. 285, 203 (1995).

[22] J. M. Lopez and F. Marques, Phys. Fluids 22, 114109 (2010).

[23] R. R. Kerswell, J. Fluid Mech. 298, 311 (1995).

[24] W. V. R. Malkus, Geophys. Astrophys. Fluid Dyn. 48, 123 (1989).

[25] C. Eloy, P. Le Gal, and S. Le Dizés, J. Fluid Mech. 476, 357 (2003).

[26] M. Le Bars, S. Le Dizés, and P. Le Gal, J. Fluid Mech. 585, 323 (2007).

[27] R. Manasseh, J. Fluid Mech. 243, 261 (1992).

[28] R. Manasseh, J. Fluid Mech. 265, 345 (1994).

[29] J. J. Kobine, J. Fluid Mech. 303, 233 (1995).

[30] J. J. Kobine, J. Fluid Mech. 319, 387 (1996).

[31] R. Lagrange, C. Eloy, F. Nadal, and P. Meunier, Phys. Fluids 20, 081701 (2008).

[32] P. Meunier, C. Eloy, R. Lagrange, and F. Nadal, J. Fluid Mech. 599, 405 (2008).

[33] R. Lagrange, P. Meunier, F. Nadal, and C. Eloy, J. Fluid Mech. 666, 104 (2011).
[34] J. Noir, M. A. Calkins, J. Cantwell, and J. M. Aurnou, Phys. Earth Planet. Inter. 182, 98 (2010).

[35] J. Boisson, C. Lamriben, L. R. M. Maas, P. P. Cortet, and F. Moisy, Phys. Fluids 24, 076602 (2012).

[36] R. R. Kerswell, J. Fluid Mech. 382, 283 (1999).

[37] D. M. Mason and R. R. Kerswell, J. Fluid Mech. 396, 73 (1999).

[38] J. M. Lopez, Phys. Fluids 8, 2605 (1996).

[39] J. M. Lopez and F. Marques, J. Fluid Mech. 687, 171 (2011).

[40] I. Mercader, O. Batiste, and A. Alonso, Comput. Fluids 39, 215 (2010)

[41] C. Panades, F. Marques, and J. M. Lopez, J. Fluid Mech. 681, 515 (2011).

[42] See Supplemental Material at http://link.aps.org/supplemental/ 10.1103/PhysRevE.89.013013 for movies showing (a) the azimuthal velocity and vorticity for $\Omega=10^{5}, \operatorname{Re}=10^{2}, \Gamma=2$, and $F=0.7071068$ over one forcing period (movie04a.avi and movie04b.avi corresponding to Fig. 4), (b) isosurfaces of the nonaxisymmetric contributions to the azimuthal vorticity of the modulated rotating wave state at $\Omega=10^{3}, \Gamma=2$, $\operatorname{Re}=2 \times 10^{3}$, and $F=0.7071068$ over one forcing period (movie10.avi corresponding to Fig. 10), (c) the azimuthal vorticity for the modulated rotating wave state at $\Omega=10^{3}$, $\Gamma=2, \operatorname{Re}=2 \times 10^{3}$, and $F=0.7071068$ over one forcing period (movie11.avi corresponding to Fig. 11), and (d) the azimuthal vorticity for $\Omega=10^{3}, \Gamma=2$, and $\operatorname{Re}=10^{2}$, using with time replaced by increasing $F$ values in the range $F \in(0,1]$ and all frames are at a phase where the displacement of the sidewall is maximum in the negative- $z$ direction (movie14.avi corresponding to Fig. 14).

[43] I. C. Walton, Proc. R. Soc. A 344, 101 (1975).

[44] H. Schlichting and K. Gersten, Boundary-Layer Theory, 8th corrected printing ed. (Springer-Verlag, Berlin, Heidelberg, 2003).

[45] C.-S. Yih, Fluid Mechanics (West River Press, Ann Arbor, MI, 1977).

[46] C. Thomas, A. P. Bassom, P. J. Blennerhassett, and C. Davies, J. Eng. Math. 68, 327 (2010).

[47] J. M. Lopez, F. Marques, A. M. Rubio, and M. Avila, Phys. Fluids 21, 114107 (2009).

[48] Y. Do, J. M. Lopez, and F. Marques, Phys. Rev. E 82, 036301 (2010).

[49] K. Zhang and X. Liao, J. Fluid Mech. 610, 425 (2008).

[50] X. Liao and K. Zhang, J. Fluid Mech. 709, 610 (2012).

[51] L. R. M. Maas, J. Fluid Mech. 437, 13 (2001).

[52] L. R. M. Maas, Int. J. Bifurcation Chaos Appl. Sci. Eng. 15, 2757 (2005).

[53] I. D. Borcia and U. Harlander, Theor. Comput. Fluid Dyn. 27, 397 (2013).

[54] A. Rabitti and L. R. M. Maas, J. Fluid Mech. 729, 445 (2013).

[55] M. Abramowicz and I. Stegun, Handbook of Mathematical Functions (Dover, New York, 1965). 